\title{
HITTING TIME STATISTICS AND EXTREME VALUE THEORY
}

\author{
ANA CRISTINA MOREIRA FREITAS, JORGE MILHAZES FREITAS, AND MIKE TODD
}

\begin{abstract}
We consider discrete time dynamical systems and show the link between Hitting Time Statistics (the distribution of the first time points land in asymptotically small sets) and Extreme Value Theory (distribution properties of the partial maximum of stochastic processes). This relation allows to study Hitting Time Statistics with tools from Extreme Value Theory, and vice versa. We apply these results to non-uniformly hyperbolic systems and prove that a multimodal map with an absolutely continuous invariant measure must satisfy the classical extreme value laws (with no extra condition on the speed of mixing, for example). We also give applications of our theory to higher dimensional examples, for which we also obtain classical extreme value laws and exponential hitting time statistics (for balls). We extend these ideas to the subsequent returns to asymptotically small sets, linking the Poisson statistics of both processes.
\end{abstract}

\section{INTRODUCTION}

In this paper we demonstrate and exploit the link between Extreme Value Laws (EVL) and the laws for the Hitting Time Statistics (HTS) for discrete time non-uniformly hyperbolic dynamical systems.

The setting is a discrete time dynamical system $(\mathcal{X}, \mathcal{B}, \mu, f)$, where $\mathcal{X}$ is a $d$-dimensional Riemannian manifold, $\mathcal{B}$ is the Borel $\sigma$-algebra, $f: \mathcal{X} \rightarrow \mathcal{X}$ is a measurable map and $\mu$ an $f$-invariant probability measure (for all $A \in \mathcal{B}$ we have $\mu\left(f^{-1}(A)\right)=\mu(A)$ ). We consider a Riemannian metric on $\mathcal{X}$ that we denote by 'dist' and for any $\zeta \in \mathcal{X}$ and $\delta>0$, we define $B_{\delta}(\zeta)=\{x \in \mathcal{X}: \operatorname{dist}(x, \zeta)<\delta\}$. Also let Leb denote Lebesgue measure on $\mathcal{X}$ and for every $A \in \mathcal{B}$ we will write $|A|:=\operatorname{Leb}(A)$. The measure $\mu$ will be an absolutely continuous invariant probability measure (acip) with density denoted by $\rho=\frac{d \mu}{d \text { Leb }}$. We will denote $\mathbb{R}^{+}:=(0, \infty)$ and $\mathbb{R}_{0}^{+}:=[0, \infty)$.

1.1. Extreme Value Laws. In this context, by EVL we mean the study of the asymptotic distribution of the partial maximum of observable random variables evaluated along the orbits of the system. To be more precise, take an observable $\varphi: \mathcal{X} \rightarrow \mathbb{R} \cup\{ \pm \infty\}$ achieving

Date: November 1, 2018.

2000 Mathematics Subject Classification. 37A50, 37C40, 60G10, 60G70, 37B20, 37D25, 37E05.

Key words and phrases. Return Time Statistics, Extreme Value Theory, Non-uniform hyperbolicity, Interval maps.

JMF is partially supported by POCI/MAT/61237/2004 and MT is supported by FCT grant SFRH/BPD/26521/2006. All three authors are supported by FCT through CMUP. 
a global maximum at $\zeta \in \mathcal{X}$ (we allow $\varphi(\zeta)=+\infty$ ) and consider the stationary stochastic process $X_{0}, X_{1}, \ldots$ given by

$$
X_{n}=\varphi \circ f^{n}, \quad \text { for each } n \in \mathbb{N} .
$$

Define the partial maximum

$$
M_{n}:=\max \left\{X_{0}, \ldots, X_{n-1}\right\} .
$$

If $\mu$ is ergodic then Birkhoff's law of large numbers says that $M_{n} \rightarrow \varphi(\zeta)$ almost surely. Similarly to central limit laws for partial sums, we are interested in knowing if there are normalising sequences $\left\{a_{n}\right\}_{n \in \mathbb{N}} \subset \mathbb{R}^{+}$and $\left\{b_{n}\right\}_{n \in \mathbb{N}} \subset \mathbb{R}$ such that

$$
\mu\left(\left\{x: a_{n}\left(M_{n}-b_{n}\right) \leq y\right\}\right)=\mu\left(\left\{x: M_{n} \leq u_{n}\right\}\right) \rightarrow H(y),
$$

for some non-degenerate distribution function (d.f.) $H$, as $n \rightarrow \infty$. Here $u_{n}:=u_{n}(y)=$ $y / a_{n}+b_{n}$ is such that

$$
n \mu\left(X_{0}>u_{n}\right) \rightarrow \tau, \text { as } n \rightarrow \infty,
$$

for some $\tau=\tau(y) \geq 0$ and in fact $H(y)=H(\tau(y))$. When this happens we say that we have an Extreme Value Law (EVL) for $M_{n}$. Note that, clearly, we must have $u_{n} \rightarrow \varphi(\zeta)$, as $n \rightarrow \infty$. We refer to an event $\left\{X_{j}>u_{n}\right\}$ as an exceedance, at time $j$, of level $u_{n}$. Classical Extreme Value Theory asserts that there are only three types of non-degenerate asymptotic distributions for the maximum of an independent and identically distributed (i.i.d.) sample under linear normalisation. They will be referred to as classical EVLs and we denote them by:

Type 1: $E V_{1}(y)=\mathrm{e}^{-\mathrm{e}^{-y}}$ for $y \in \mathbb{R}$; this is also known as the Gumbel extreme value distribution (e.v.d.).

Type 2: $E V_{2}(y)=\mathrm{e}^{-y^{-\alpha}}$, for $y>0, E V_{2}(y)=0$, otherwise, where $\alpha>0$ is a parameter; this family of d.f.s is known as the Fréchet e.v.d.

Type 3: $E V_{3}(y)=\mathrm{e}^{-(-y)^{\alpha}}$, for $y \leq 0, E V_{3}(y)=1$, otherwise, where $\alpha>0$ is a parameter; this family of d.f.s is known as the Weibull e.v.d.

The same limit laws apply to stationary stochastic processes, under certain conditions on the dependence structure, which allow the reduction to the independent case. With this in mind, to a given stochastic process $X_{0}, X_{1}, \ldots$ we associate an i.i.d. sequence $Y_{0}, Y_{1}, \ldots$, whose d.f. is the same as that of $X_{0}$, and whose partial maximum we define as

$$
\hat{M}_{n}:=\max \left\{Y_{0}, \ldots, Y_{n-1}\right\} .
$$

In the i.i.d. setting, the convergence in (1.4) is equivalent to

$$
\mu\left(\hat{M}_{n} \leq u_{n}\right) \rightarrow \mathrm{e}^{-\tau}, \text { as } n \rightarrow \infty .
$$

This is the content of [LLR, Theorem 1.5.1] and depending on the type of limit law that applies, we have that $\tau=\tau(y)$ is of one of the following three types: $\tau_{1}(y)=\mathrm{e}^{-y}$ for $y \in \mathbb{R}$, $\tau_{2}(y)=y^{-\alpha}$ for $y>0$, and $\tau_{3}(y)=(-y)^{\alpha}$ for $y \leq 0$.

In the dependent context, the general strategy is to prove that if $X_{0}, X_{1}, \ldots$ satisfies some conditions, then the same limit law for $\hat{M}_{n}$ applies to $M_{n}$ with the same normalising 
sequences $\left\{a_{n}\right\}_{n \in \mathbb{N}}$ and $\left\{b_{n}\right\}_{n \in \mathbb{N}}$. Following [LLR] we refer to these conditions as $D\left(u_{n}\right)$ and $D^{\prime}\left(u_{n}\right)$, where $u_{n}$ is the sequence of thresholds appearing in (1.3). Both conditions impose some sort of independence but while $D\left(u_{n}\right)$ acts on the long range, $D^{\prime}\left(u_{n}\right)$ is a short range requirement.

The original condition $D\left(u_{n}\right)$ from [LLR], which we will denote by $D_{1}\left(u_{n}\right)$, is a type of uniform mixing requirement specially adapted to Extreme Value Theory. Let $F_{i_{1}, \ldots, i_{n}}\left(x_{1}, \ldots, x_{n}\right)$ denote the joint d.f. of $X_{i_{1}}, \ldots, X_{i_{n}}$, and set $F_{i_{1}, \ldots, i_{n}}(u)=F_{i_{1}, \ldots, i_{n}}(u, \ldots, u)$.

Condition $\left(D_{1}\left(u_{n}\right)\right)$. We say that $D_{1}\left(u_{n}\right)$ holds for the sequence $X_{0}, X_{1}, \ldots$ if for any integers $i_{1}<\ldots<i_{p}$ and $j_{1}<\ldots<j_{k}$ for which $j_{1}-i_{p}>m$, and any large $n \in \mathbb{N}$,

$$
\left|F_{i_{1}, \ldots, i_{p}, j_{1}, \ldots, j_{k}}\left(u_{n}\right)-F_{i_{1}, \ldots, i_{p}}\left(u_{n}\right) F_{j_{1}, \ldots, j_{k}}\left(u_{n}\right)\right| \leq \gamma(n, m),
$$

where $\gamma\left(n, m_{n}\right) \underset{n \rightarrow \infty}{\longrightarrow} 0$, for some sequence $m_{n}=o(n)$.

Since usually the information concerning mixing rates of the systems is known through decay of correlations, in FF2] we proposed a weaker version, which we will denote by $D_{2}\left(u_{n}\right)$, which still allows us to relate the distributions of $\hat{M}_{n}$ and $M_{n}$. The advantage is that it follows immediately from sufficiently fast decay of correlations for observables which are of bounded variation or Hölder continuous (see [FF2, Section 2] and Lemma 6.6).

Condition $\left(D_{2}\left(u_{n}\right)\right)$. We say that $D_{2}\left(u_{n}\right)$ holds for the sequence $X_{0}, X_{1}, \ldots$ if for any integers $\ell, t$ and $n$

$$
\left|\mu\left(\left\{X_{0}>u_{n}\right\} \cap\left\{\max \left\{X_{t}, \ldots, X_{t+\ell-1}\right\} \leq u_{n}\right\}\right)-\mu\left(\left\{X_{0}>u_{n}\right\}\right) \mu\left(\left\{M_{\ell} \leq u_{n}\right\}\right)\right| \leq \gamma(n, t),
$$

where $\gamma(n, t)$ is nonincreasing in $t$ for each $n$ and $n \gamma\left(n, t_{n}\right) \rightarrow 0$ as $n \rightarrow \infty$ for some sequence $t_{n}=o(n)$.

By (1.4), the sequence $u_{n}$ is such that the average number of exceedances in the time interval $\{0, \ldots,\lfloor n / k\rfloor\}$ is approximately $\tau / k$, which goes to zero as $k \rightarrow \infty$. However, the exceedances may have a tendency to be concentrated in the time period following the first exceedance at time 0 . To avoid this we introduce:

Condition $\left(D^{\prime}\left(u_{n}\right)\right)$. We say that $D^{\prime}\left(u_{n}\right)$ holds for the sequence $X_{0}, X_{1}, \ldots$ if

$$
\lim _{k \rightarrow \infty} \limsup _{n \rightarrow \infty} n \sum_{j=1}^{\lfloor n / k\rfloor} \mu\left(\left\{X_{0}>u_{n}\right\} \cap\left\{X_{j}>u_{n}\right\}\right)=0 .
$$

This guarantees that the exceedances should appear scattered through the time period $\{0, \ldots, n-1\}$.

The main result in [FF2, Theorem 1] states that if $D_{2}\left(u_{n}\right)$ and $D^{\prime}\left(u_{n}\right)$ hold for the process $X_{0}, X_{1}, \ldots$ and for a sequence of levels satisfying (1.4), then the following limits exist, and

$$
\lim _{n \rightarrow \infty} \mu\left(\hat{M}_{n} \leq u_{n}\right)=\lim _{n \rightarrow \infty} \mu\left(M_{n} \leq u_{n}\right) .
$$


The above statement remains true if we replace $D_{2}\left(u_{n}\right)$ by $D_{1}\left(u_{n}\right)$ (see [LLR, Theorem 3.5.2]).

We assume that the observable $\varphi: \mathcal{X} \rightarrow \mathbb{R} \cup\{+\infty\}$ is of the form

$$
\varphi(x)=g(\operatorname{dist}(x, \zeta))
$$

where $\zeta$ is a chosen point in the phase space $\mathcal{X}$ and the function $g:[0,+\infty) \rightarrow \mathbb{R} \cup\{+\infty\}$ is such that 0 is a global maximum $(g(0)$ may be $+\infty) ; g$ is a strictly decreasing bijection $g: V \rightarrow W$ in a neighbourhood $V$ of 0 ; and has one of the following three types of behaviour:

Type 1: there exists some strictly positive function $p: W \rightarrow \mathbb{R}$ such that for all $y \in \mathbb{R}$

$$
\lim _{s \rightarrow g_{1}(0)} \frac{g_{1}^{-1}(s+y p(s))}{g_{1}^{-1}(s)}=\mathrm{e}^{-y}
$$

Type 2: $g_{2}(0)=+\infty$ and there exists $\beta>0$ such that for all $y>0$

$$
\lim _{s \rightarrow+\infty} \frac{g_{2}^{-1}(s y)}{g_{2}^{-1}(s)}=y^{-\beta}
$$

Type 3: $g_{3}(0)=D<+\infty$ and there exists $\gamma>0$ such that for all $y>0$

$$
\lim _{s \rightarrow 0} \frac{g_{3}^{-1}(D-s y)}{g_{3}^{-1}(D-s)}=y^{\gamma} .
$$

Examples of each one of the three types are as follows: $g_{1}(x)=-\log x$ (in this case (1.10) is easily verified with $p \equiv 1$ ), $g_{2}(x)=x^{-1 / \alpha}$ for some $\alpha>0$ (condition (1.11) is verified with $\beta=\alpha$ ) and $g_{3}(x)=D-x^{1 / \alpha}$ for some $D \in \mathbb{R}$ and $\alpha>0$ (condition (1.12) is verified with $\gamma=\alpha$ ).

Remark 1. Let the d.f. $F$ be given by $F(u)=\mu\left(X_{0} \leq u\right)$ and set $u_{F}=\sup \{y: F(y)<1\}$. Observe that if at time $j \in \mathbb{N}$ we have an exceedance of the level $u$ (sufficiently large), i.e., $X_{j}(x)>u$, then we have an entrance of the orbit of $x$ into the ball $B_{g^{-1}(u)}(\zeta)$ of radius $g^{-1}(u)$ around $\zeta$, at time $j$. This means that the behaviour of the tail of $F$, i.e., the behaviour of $1-F(u)$ as $u \rightarrow u_{F}$ is determined by $g^{-1}$, if we assume that Lebesgue's Differentiation Theorem holds for $\zeta$, since in that case $1-F(u) \sim \rho(\zeta)\left|B_{g^{-1}(u)}(\zeta)\right|$, where $\rho(\zeta)=\frac{d \mu}{d \operatorname{Leb}}(\zeta)$. From classical Extreme Value Theory we know that the behaviour of the tail determines the limit law for partial maximums of i.i.d. sequences and vice-versa. The above conditions are just the translation in terms of the shape of $g^{-1}$, of the sufficient and necessary conditions on the tail of $F$ of [LLR, Theorem 1.6.2], in order to exist a non-degenerate limit distribution for $\hat{M}_{n}$. In fact, if some $E V_{i}$ applies to $\hat{M}_{n}$, for some $i \in\{1,2,3\}$, then $g$ must be of type $g_{i}$.

As can be seen from the definitions of $D_{2}\left(u_{n}\right)$ and $D^{\prime}\left(u_{n}\right)$, proving EVLs for absolutely continuous invariant measures for uniformly expanding dynamical systems is straightforward. The study of EVLs for non-uniformly hyperbolic dynamical systems has been addressed in the papers [Col2 and [FF1. 
In [Col2], Collet considered non-uniformly hyperbolic $C^{2}$ maps of the interval which admit an acip $\mu$, with exponential decay of correlations and obtained a Gumbel EVL for observables of type $g_{1}$ (actually he took $g_{1}(x)=-\log x$ ), achieving a global maximum at $\mu$-a.e. $\zeta$ in the phase space. We remark that neither the critical points nor its orbits were included in this full $\mu$-measure set of points $\zeta$.

In [FF1] the quadratic maps $f_{a}(x)=1-a x^{2}$ on $I=[-1,1]$ were considered, with $a \in \mathcal{B} C$, where $\mathcal{B C}$ is the Benedicks-Carleson parameter set introduced in $[\mathrm{BC}]$. For each map $f_{a}$ with $a \in \mathcal{B} C$, a Weibull EVL was obtained for observables of type $g_{3}$ achieving a maximum either at the critical point or at the critical value.

1.2. Hitting Time Statistics. We next turn to Hitting Time Statistics for the dynamical system $(\mathcal{X}, \mathcal{B}, f, \mu)$. For a set $A \subset \mathcal{X}$ we let $r_{A}(y)$ denote the first hitting time to $A$ of the point $y$. That is, the first time $j \geq 1$ so that $f^{j}(y) \in A$. We will be interested in the fluctuations of this functions as the set $A$ shrinks. Firstly we consider the Return Time Statistics (RTS) of this system. Let $\mu_{A}$ denote the conditional measure on A, i.e., $\mu_{A}:=$ $\frac{\left.\mu\right|_{A}}{\mu(A)}$. By Kac's Lemma, the expected value of $r_{A}$ with respect to $\mu$ is $\int_{A} r_{A} d \mu_{A}=1 / \mu(A)$. So in studying the fluctuations of $r_{A}$ on $A$, the relevant normalising factor is $1 / \mu(A)$. Given a sequence of sets $\left\{U_{n}\right\}_{n \in \mathbb{N}}$ so that $\mu\left(U_{n}\right) \rightarrow 0$, the system has Return Time Statistics $G(t)$ for $\left\{U_{n}\right\}_{n \in \mathbb{N}}$ if for all $t \geq 0$ the following limit exists and equals $G(t)$ :

$$
\lim _{n \rightarrow \infty} \mu_{U_{n}}\left(r_{U_{n}} \geq \frac{t}{\mu\left(U_{n}\right)}\right) .
$$

We say that $(\mathcal{X}, f, \mu)$ has Return Time Statistics $G(t)$ to balls at $\zeta$ if for any sequence $\left\{\delta_{n}\right\}_{n \in \mathbb{N}} \subset \mathbb{R}^{+}$such that $\delta_{n} \rightarrow 0$ as $n \rightarrow \infty$ we have RTS $G(t)$ for $U_{n}=B_{\delta_{n}}(\zeta)$.

If we study $r_{A}$ defined on the whole of $\mathcal{X}$, i.e., not simply restricted to $A$, we are studying the Hitting Time Statistics. Note that we will use the same normalising factor $1 / \mu(A)$ in this case. Analogously to the above, given a sequence of sets $\left\{U_{n}\right\}_{n \in \mathbb{N}}$ so that $\mu\left(U_{n}\right) \rightarrow 0$, the system has Hitting Time Statistics $G(t)$ for $\left\{U_{n}\right\}_{n \in \mathbb{N}}$ if for all $t \geq 0$ the following limit is defined and equals $G(t)$ :

$$
\lim _{n \rightarrow \infty} \mu\left(r_{U_{n}} \geq \frac{t}{\mu\left(U_{n}\right)}\right) .
$$

HTS to balls at a point $\zeta$ is defined analogously to RTS to balls. In [HLV], it was shown that the limit for the HTS defined in (1.14) exists if and only if the limit for the analogous RTS defined in (1.13) exists. Moreover, they show that the HTS distribution exists and is exponential (i.e., $G(t)=\mathrm{e}^{-t}$ ) if and only if the RTS distribution exists and is exponential.

For many mixing systems it is known that the HTS are exponential. For example, this was shown for Axiom A diffeomorphisms in [H], transitive Markov chains in [Pi] and uniformly expanding maps of the interval in [Col1]. Note that in these papers the authors were also interested in the (Poisson) statistics of subsequent returns to some shrinking sets. For various results on some systems with some strong hyperbolicity properties see also e.g. [Ab, $\mathrm{C} 1, \mathrm{AG}$. 
For non-uniformly hyperbolic systems less is known. A major breakthrough in the study of HTS/RTS for non-uniformly hyperbolic maps was made in [HSV], where they gave a set of conditions which, when satisfied, imply exponential RTS to cylinders and/or balls. Their principal application was to maps of the interval with an indifferent fixed point. They also provided similar conditions to imply (Poisson) laws for the subsequent visits of points to shrinking sets. (See Section 5).

Another important paper in this direction was BSTV], in which they showed that the RTS for a map are the same as the RTS for the first return map. (The first return map to a set $U \subset \mathcal{X}$ is the map $F=f^{r_{U}}$.) Since it is often the case that the first return maps for non-uniformly hyperbolic dynamical systems are much better behaved (possibly hyperbolic) than the original system, this provided an extremely useful tool in this theory. For example, they proved that if $f: I \rightarrow I$ is a unimodal map for which the critical point is nowhere dense, and for which an acip $\mu$ exists, then the relevant first return systems $\left(U, F, \mu_{U}\right)$ have a 'Rychlik' property. They then showed that such systems, studied in $[\mathbf{R}$, must have exponential RTS, and hence the original system $(I, f, \mu)$ also has exponential RTS (to balls around $\mu$-a.e. point).

The presence of a recurrent critical point means that the first return map itself will not satisfy this Rychlik property. To overcome this problem in [BV] special induced maps, $(U, F)$, were used, where for $x \in U$ we have $F(x)=f^{\operatorname{ind}(x)}(x)$ for some inducing time $\operatorname{ind}(x) \in \mathbb{N}$ that is not necessarily the first return time of $x$ to $U$. The fact that these particular maps can be seen as first return maps in the canonical Markov extension, the 'Hofbauer tower', meant that they were still able to exploit the main result of [BSTV] to get exponential RTS around $\mu$-a.e. point for unimodal maps $f: I \rightarrow I$ with an acip $\mu$ as long as $f$ satisfies a polynomial growth condition along the critical orbit. In BT] this result was improved to include any multimodal map with an acip, irrespective of the growth along the critical orbits, and of the speed of mixing.

We would like to remark that in the case of partially hyperbolic dynamical systems, Do] proved exponential RTS, using techniques similar to [Pi]. In fact the theory there also covers the (Poisson) statistics of subsequent returns to shrinking sets of balls. These statistics were also considered for toral automorphisms, using a different method, in [DGS].

We note that for dynamical systems $(\mathcal{X}, \mathcal{B}, f, \mu)$ where $\mu$ is an equilibrium state, the RTS/HTS to the dynamically defined cylinders are often well understood, see for example AG]. However, for non-uniformly hyperbolic dynamical systems it is not always possible to go from these strong results to the corresponding results for balls. We would like to emphasise that in this paper we focus on the HTS to balls, rather than cylinders.

1.3. Main Results. Our first main result, which obtains EVLs from HTS, is the following.

Theorem 1. Let $(\mathcal{X}, \mathcal{B}, \mu, f)$ be a dynamical system where $\mu$ is an acip, and consider $\zeta \in \mathcal{X}$ for which Lebesgue's Differentiation Theorem holds. 
- If we have HTS to balls centred on $\zeta \in \mathcal{X}$, then we have an EVL for $M_{n}$ which applies to the observables (1.9) achieving a maximum at $\zeta$.

- If we have exponential HTS $\left(G(t)=e^{-t}\right)$ to balls at $\zeta \in \mathcal{X}$, then we have an EVL for $M_{n}$ which coincides with that of $\hat{M}_{n}$ (meaning that (1.8) holds). In particular, this EVL must be one of the 3 classical types. Moreover, if $g$ is of type $g_{i}$, for some $i \in\{1,2,3\}$, then we have an $E V L$ for $M_{n}$ of type $E V_{i}$.

We next define a class of multimodal interval maps $f: I \rightarrow I$. We denote the finite set of critical points by Crit. We say that $c \in$ Crit is non-flat if there exists a diffeomorphism $\psi_{c}$ : $\mathbb{R} \rightarrow \mathbb{R}$ with $\psi_{c}(0)=0$ and $1<\ell_{c}<\infty$ such that for $x$ close to $c, f(x)=f(c) \pm\left|\psi_{c}(x-c)\right|^{\ell_{c}}$. The value of $\ell_{c}$ is known as the critical order of $c$. Let

$$
N F^{k}:=\left\{f: I \rightarrow I: f \text { is } C^{k} \text {, each } c \in \text { Crit is non-flat and } \inf _{f^{n}(p)=p}\left|D f^{n}(p)\right|>1\right\} .
$$

The following is a simple corollary of Theorem 1 and [BT, Theorem 3]. It generalises the result of Collet in [Col2 from unimodal maps with exponential growth on the critical point to multimodal maps where we only need to know that there is an acip.

Corollary 1. Suppose that $f \in N F^{2}$ and $f$ has an acip $\mu$. Then $(I, f, \mu)$ has an $E V L$ for $M_{n}$ which coincides with that of $\hat{M}_{n}$, and this holds for $\mu$-a.e. $\zeta \in \mathcal{X}$ fixed at the choice of the observable in (1.9). Moreover, the EVL is of type $E V_{i}$ when the observables are of type $g_{i}$, for each $i \in\{1,2,3\}$.

Now, we state a result in the other direction, i.e., we show how to get HTS from EVLs.

Theorem 2. Let $(\mathcal{X}, \mathcal{B}, \mu, f)$ be a dynamical system where $\mu$ is an acip and consider $\zeta \in \mathcal{X}$ for which Lebesgue's Differentiation Theorem holds.

- If we have an EVL for $M_{n}$ which applies to the observables (1.9) achieving a maximum at $\zeta \in \mathcal{X}$ then we have HTS to balls at $\zeta$.

- If we have an $E V L$ for $M_{n}$ which coincides with that of $\hat{M}_{n}$, then we have exponential $\operatorname{HTS}\left(G(t)=e^{-t}\right)$ to balls at $\zeta$.

The following is immediate by the above and [FF2, Theorem 1] (see (1.8)).

Corollary 2. Let $(\mathcal{X}, \mathcal{B}, \mu, f)$ be a dynamical system where $\mu$ is an acip and consider $\zeta \in \mathcal{X}$ for which Lebesgue's Differentiation Theorem holds. If $D_{2}\left(u_{n}\right)$ (or $D_{1}\left(u_{n}\right)$ ) and $D^{\prime}\left(u_{n}\right)$ hold for a stochastic process $X_{0}, X_{1}, \ldots$ defined by (1.1) and (1.9), where $u_{n}$ is a sequence of levels satisfying (1.4), then we have exponential HTS to balls at $\zeta$.

The following is an immediate corollary of Theorem 2 and the main theorem of [FF1].

Corollary 3. For every Benedicks-Carleson quadratic map $f_{a}$ (with $a \in \mathcal{B C}$ ) we have exponential HTS to balls around the critical point or the critical value. 
The next result is a byproduct of Theorems 1, 2 and the fact that under $D_{1}\left(u_{n}\right)$ the only possible limit laws for partial maximums are the classical $E V_{i}$ for $i \in\{1,2,3\}$. Since this is not as immediate as the other corollaries, we include a short proof in Section 2 .

Corollary 4. Let $(\mathcal{X}, \mathcal{B}, \mu, f)$ be a dynamical system $\mu$ is an acip and consider $\zeta \in \mathcal{X}$ for which Lebesgue's Differentiation Theorem holds. If $D_{1}\left(u_{n}\right)$ holds for a stochastic process $X_{0}, X_{1}, \ldots$ defined by (1.1) and (1.9), where $u_{n}$ is a sequence of levels satisfying (1.4), then the only possible HTS to balls around $\zeta$ are of exponential type, meaning that, there is $\theta>0$ such that $G(t)=e^{-\theta t}$.

Note that for this corollary to be non-trivial, we must assume that there exists a distribution for HTS. This may not always be the case. For example, in $\mathrm{CF}$, C2 it was shown that for certain circle diffeomorphisms there are sequences of intervals $\left\{U_{n}\right\}_{n \in \mathbb{N}},\left\{V_{n}\right\}_{n \in \mathbb{N}}$ which both shrink to the same point $\zeta$, but yield different HTS laws. Note that in these cases $D_{1}\left(u_{n}\right)$ also fails.

As we have already mentioned, Corollary 1 generalises the result of Collet in Col2, which was for $C^{2}$ non-uniformly hyperbolic maps of the interval (admitting a Young tower). However, a close look to Collet's arguments allows us to conclude that his result still prevails in higher dimensions. In fact, one can show that if we consider non-uniformly expanding maps (in any finite dimensional compact manifold), admitting a so-called Young tower with exponential return times to the base, then for any sequence of r.v. $X_{0}, X_{1}, \ldots$, defined as in (1.1) and for a sequence of levels $u_{n}$ such that $n \mu\left(X_{0}>u_{n}\right) \rightarrow \tau>0$, conditions $D_{2}\left(u_{n}\right)$ and $D^{\prime}\left(u_{n}\right)$ hold. This means that by the above theorems, we can prove both EVLs and HTS for these maps. Due to numerous definitions required for that setting, we leave both the theorems and the proofs on this subject to Section 6 .

Theorems 11 and 2 give us new tools to investigate the recurrence of dynamical systems, principally by allowing us to use the wealth of theory for HTS which has been developed in recent years to prove EVLs. We note that in Corollary 1, the dynamical systems involved need not have any fast rate of decay of correlations at all. Indeed, a priori the relevant system may only have summable decay of correlations. As in Section 6 where we consider higher dimensional maps admitting Young towers, there are situations where it is actually easier to check conditions like $D_{2}\left(u_{n}\right)$ and $D^{\prime}\left(u_{n}\right)$ in order to get laws for HTS. In fact, to our knowledge, exponential HTS to balls have never been proved before for higher dimensional non-uniformly expanding systems: in such cases, inducing schemes with the nice properties of one-dimensional dynamics are much harder to find. Also the dynamical systems we present in this paper should provide models which can be used in investigating Extreme Value Theory both analytically and numerically. Namely, the simple fact that we get EVLs from deterministic models may be an extra advantage for numerical simulation since there is no need to generate random numbers. This means that this theory may reveal very useful for testing GEV (Generalised Extreme Value distribution) fitting for data corresponding to phenomena for which there is an underlying deterministic model. 
The next question that arises is: what about subsequent visits to $U_{n}$ or subsequent exceedances of the level $u_{n}$ ? Namely, we are interested in the point processes associated to the instants of occurrence of returns to $U_{n}$ and exceedances of the level $u_{n}$. If we have either exponential HTS or a classical EVL then time between hits or exceedances is exponentially distributed. This means that we should expect a Poisson limit for the point processes. We show in Section 3 that the relation between HTS and EVL does indeed extend to the laws for the subsequent visits/exceedances (we postpone the precise definitions and results to Sections 3, 4 and 51). More precisely, we show that the point process of hitting times has a Poisson limit if and only if the point process of exceedances has a Poisson limit. We next discuss how to obtain a Poisson law in these two different contexts. In Section 4 we give conditions which guarantee a Poisson limit for the point process of exceedance times. This part of the paper can be seen as a generalisation of [FF2]. Moreover, we show that these conditions can be verified in the settings from [Col2, FF1, leading to Poisson statistics for both point processes for the systems considered. In Section 5 we show that in many cases for multimodal maps it can be shown that the HTS behave asymptotically as a Poisson distribution.

Throughout this paper the notation $A_{n} \sim B_{n}$ means that $\lim _{n \rightarrow \infty} \frac{A_{n}}{B_{n}}=1$. Also, if $\left\{\delta_{n}\right\}_{n \in \mathbb{N}} \subset \mathbb{R}^{+}$has $\delta_{n} \rightarrow 0$ as $n \rightarrow \infty$, then for each $\zeta \in \mathcal{X}$, let $\kappa \in(0, \infty)$ be such that $\left|B_{\delta_{n}}(\zeta)\right| \sim \kappa \cdot \delta_{n}^{d}$. Let $x \in \mathbb{R}$. We denote the integer part of $x$ by $\lfloor x\rfloor$ and define $\lceil x\rceil:=x$ if $x=\lfloor x\rfloor$, and $\lceil x\rceil:=\lfloor x\rfloor+1$ otherwise.

Acknowledgements. We would like to thank J.F. Alves for useful suggestions regarding the example of a non-uniformly expanding system given in Section 6.2.

\section{Proofs of our Results on HTS ANd EVL}

In this section we prove Theorems 1, 2] and Corollary 4 .

Proof of Theorem 11. Let $\rho(\zeta)=\frac{d \mu}{d \mathrm{Leb}}(\zeta) \in \mathbb{R}_{0}^{+}$and set

$$
\begin{array}{ll}
u_{n}=g_{1}\left((\kappa \rho(\zeta) n)^{-1 / d}\right)+p\left(g_{1}\left((\kappa \rho(\zeta) n)^{-1 / d}\right)\right) \frac{y}{d}, & \text { for } y \in \mathbb{R}, \text { for type } g_{1} ; \\
u_{n}=g_{2}\left((\kappa \rho(\zeta) n)^{-1 / d}\right) y, & \text { for } y>0, \text { for type } g_{2} ; \\
u_{n}=D-\left(D-g_{3}\left((\kappa \rho(\zeta) n)^{-1 / d}\right)\right)(-y), & \text { for } y<0, \text { for type } g_{3} .
\end{array}
$$

Note that, for $n$ sufficiently large

$$
\begin{aligned}
& \left\{x: M_{n}(x) \leq u_{n}\right\}=\bigcap_{j=0}^{n-1}\left\{x: X_{j}(x) \leq u_{n}\right\}=\bigcap_{j=0}^{n-1}\left\{x: g\left(\operatorname{dist}\left(f^{j}(x), \zeta\right)\right) \leq u_{n}\right\} \\
& =\bigcap_{j=0}^{n-1}\left\{x: \operatorname{dist}\left(f^{j}(x), \zeta\right) \geq g^{-1}\left(u_{n}\right)\right\}=\left\{x: r_{B_{g^{-1}\left(u_{n}\right)}(\zeta)}(x) \geq n\right\}
\end{aligned}
$$


Now, observe that (1.10), (1.11) and (1.12) imply

$$
\begin{aligned}
g_{1}^{-1}\left(u_{n}\right) & =g_{1}^{-1}\left[g_{1}\left((\kappa \rho(\zeta) n)^{-1 / d}\right)+p\left(g_{1}\left((\kappa \rho(\zeta) n)^{-1 / d}\right)\right) \frac{y}{d}\right] \\
& \sim g_{1}^{-1}\left[g_{1}\left((\kappa \rho(\zeta) n)^{-1 / d}\right)\right] \mathrm{e}^{-y / d}=\left(\frac{\mathrm{e}^{-y}}{\kappa \rho(\zeta) n}\right)^{1 / d} ; \\
g_{2}^{-1}\left(u_{n}\right) & =g_{2}^{-1}\left[g_{2}\left((\kappa \rho(\zeta) n)^{-1 / d}\right) y\right] \sim g_{2}^{-1}\left[g_{2}\left((\kappa \rho(\zeta) n)^{-1 / d}\right)\right] y^{-\beta}=\left(\frac{y^{-\beta d}}{\kappa \rho(\zeta) n}\right)^{1 / d} ; \\
g_{3}^{-1}\left(u_{n}\right) & =g_{3}^{-1}\left[D-\left(D-g_{3}\left((\kappa \rho(\zeta) n)^{-1 / d}\right)\right)(-y)\right] \\
& \sim g_{3}^{-1}\left[D-\left(D-g_{3}\left((\kappa \rho(\zeta) n)^{-1 / d}\right)\right)\right](-y)^{\gamma}=\left(\frac{(-y)^{\gamma d}}{\kappa \rho(\zeta) n}\right)^{1 / d} .
\end{aligned}
$$

Thus, we may write

$$
g^{-1}\left(u_{n}\right) \sim\left(\frac{\tau(y)}{\kappa \rho(\zeta) n}\right)^{1 / d}
$$

meaning that

$$
g_{i}^{-1}\left(u_{n}\right) \sim\left(\frac{\tau_{i}(y)}{\kappa \rho(\zeta) n}\right)^{1 / d}, \quad \forall i \in\{1,2,3\}
$$

where $\tau_{1}(y)=\mathrm{e}^{-y}$ for $y \in \mathbb{R}, \tau_{2}(y)=y^{-\beta d}$ for $y>0$, and $\tau_{3}(y)=(-y)^{\gamma d}$ for $y<0$.

Since Lebesgue's Differentiation Theorem holds for $\zeta \in \mathcal{X}$, we have $\frac{\mu\left(B_{\delta}(\zeta)\right)}{\left|B_{\delta}(\zeta)\right|} \rightarrow \rho(\zeta)$ as $\delta \rightarrow 0$. Consequently, since it is obvious that $g^{-1}\left(u_{n}\right) \rightarrow 0$ as $n \rightarrow \infty$, then

$$
\mu\left(B_{g^{-1}\left(u_{n}\right)}(\zeta)\right) \sim \rho(\zeta)\left|B_{g^{-1}\left(u_{n}\right)}(\zeta)\right| \sim \rho(\zeta) \kappa\left(g^{-1}\left(u_{n}\right)\right)^{d}=\rho(\zeta) \kappa \frac{\tau(y)}{\kappa \rho(\zeta) n}=\frac{\tau(y)}{n} .
$$

Thus, we have

$$
n \sim \frac{\tau(y)}{\mu\left(B_{g^{-1}\left(u_{n}\right)}(\zeta)\right)}
$$

Now, we claim that using (2.1) and (2.2), we have

$$
\begin{aligned}
\lim _{n \rightarrow \infty} \mu\left(\left\{x: M_{n}(x) \leq u_{n}\right\}\right) & =\lim _{n \rightarrow \infty} \mu\left(\left\{x: r_{B_{g^{-1}\left(u_{n}\right)}(\zeta)}(x) \geq \frac{\tau(y)}{\mu\left(B_{g^{-1}\left(u_{n}\right)}(\zeta)\right)}\right\}\right) \\
& =G(\tau(y)),
\end{aligned}
$$

which gives the first part of the theorem. 
To see that (2.3) holds, observe that by (2.1) and (2.2) we have

$$
\begin{aligned}
\left|\mu\left(\left\{M_{n} \leq u_{n}\right\}\right)-\mu\left(\left\{r_{B_{g^{-1}\left(u_{n}\right)}(\zeta)} \geq \frac{\tau(y)}{\mu\left(B_{g^{-1}\left(u_{n}\right)}(\zeta)\right)}\right\}\right)\right| \\
\quad=\left|\mu\left(\left\{r_{B_{g^{-1}\left(u_{n}\right)}(\zeta)} \geq n\right\}\right)-\mu\left(\left\{r_{B_{g^{-1}\left(u_{n}\right)}(\zeta)} \geq\left(1+\varepsilon_{n}\right) n\right\}\right)\right|,
\end{aligned}
$$

where $\left\{\varepsilon_{n}\right\}_{n \in \mathbb{N}}$ is such that $\varepsilon_{n} \rightarrow 0$ as $n \rightarrow \infty$. Since we have

$$
\left\{r_{B_{g^{-1}\left(u_{n}\right)}(\zeta)} \geq m\right\} \backslash\left\{r_{B_{g^{-1}\left(u_{n}\right)}(\zeta)} \geq m+k\right\} \subset \bigcup_{j=m}^{m+k-1} f^{-j}\left(B_{g^{-1}\left(u_{n}\right)}(\zeta)\right), \forall m, k \in \mathbb{N},
$$

it follows by stationarity that

$$
\begin{aligned}
\left|\mu\left(\left\{r_{B_{g^{-1}\left(u_{n}\right)}(\zeta)} \geq n\right\}\right)-\mu\left(\left\{r_{B_{g^{-1}\left(u_{n}\right)}(\zeta)} \geq\left(1+\varepsilon_{n}\right) n\right\}\right)\right| \\
\quad \leq\left|\varepsilon_{n}\right| n \mu\left(B_{g^{-1}\left(u_{n}\right)}(\zeta)\right) \sim\left|\varepsilon_{n}\right| \tau \rightarrow 0,
\end{aligned}
$$

as $n \rightarrow \infty$, completing the proof of (2.3).

Next we will use the exponential HTS hypothesis, that is $G(t)=\mathrm{e}^{-t}$, to show the second part of the theorem.

Under the exponential HTS assumption, by (2.4) it follows immediately that $\lim _{n \rightarrow \infty} \mu(\{x$ : $\left.\left.M_{n}(x) \leq u_{n}\right\}\right)=\mathrm{e}^{-\tau(y)}$. Now, recalling that in the i.i.d setting (1.4) is equivalent to (1.6) then we also have $\lim _{n \rightarrow \infty} \mu\left(\left\{x: \hat{M}_{n}(x) \leq u_{n}\right\}\right)=\mathrm{e}^{-\tau(y)}$. As explained in the introduction, this means that $G(\tau)$ must be of the three classical types.

It remains to show that if the observable is of type $g_{i}$ then the EVL that applies to $M_{n}$ is of type $E V_{i}$, for each $i \in\{1,2,3\}$.

Type $g_{1}$ : In this case we have $\mathrm{e}^{-\tau_{1}(y)}=\mathrm{e}^{-\mathrm{e}^{-y}}$, for all $y \in \mathbb{R}$, that corresponds to the Gumbel e.v.d. and so we have an EVL for $M_{n}$ of type $E V_{1}$.

Type $g_{2}$ : We obtain $\mathrm{e}^{-\tau_{2}(y)}=\mathrm{e}^{-y^{-\beta d}}$ for $y>0$. To conclude that in this case we have the Fréchet e.v.d. with parameter $\beta d$, we only have to check that for $y \leq 0, \mu\left(\left\{x: M_{n}(x) \leq\right.\right.$ $\left.\left.u_{n}\right\}\right)=0$. Since $g_{2}\left((\kappa \rho(\zeta) n)^{-1 / d}\right)>0$ (for all large $n$ ) and

$$
\mu\left(\left\{x: M_{n}(x) \leq u_{n}\right\}\right)=\mu\left(\left\{x: M_{n}(x) \leq g_{2}\left((\kappa \rho(\zeta) n)^{-1 / d}\right) y\right\}\right) \rightarrow \mathrm{e}^{-y^{-\beta d}}
$$

as $n \rightarrow \infty$. Letting $y \downarrow 0$, it follows that $\mu\left(\left\{x: M_{n}(x) \leq 0\right\}\right) \rightarrow 0$, and, for $y<0$,

$\mu\left(\left\{x: M_{n}(x) \leq u_{n}\right\}\right)=\mu\left(\left\{x: M_{n}(x) \leq g_{2}\left((\kappa \rho(\zeta) n)^{-1 / d}\right) y\right\}\right) \leq \mu\left(\left\{x: M_{n}(x) \leq 0\right\}\right) \rightarrow 0$.

So, we have, in this case, an EVL for $M_{n}$ of type $E V_{2}$.

Type $g_{3}$ : For $y<0$, we have $\mathrm{e}^{-\tau_{3}(y)}=\mathrm{e}^{-(-y)^{\gamma d}}$. To conclude that in this case we have the Weibull e.v.d. with parameter $\gamma d$, we only need to check that for $y \geq 0, \mu\left(\left\{x: M_{n}(x) \leq\right.\right.$ 
$\left.\left.u_{n}\right\}\right)=1$. In fact, for $y \geq 0$, since $D-g_{3}\left((\kappa \rho(\zeta) n)^{-1 / d}\right)>0$, we have

$$
\begin{aligned}
\mu\left(\left\{x: M_{n}(x) \leq u_{n}\right\}\right) & =\mu\left(\left\{x: M_{n}(x) \leq\left(D-g_{3}\left((\kappa \rho(\zeta) n)^{-1 / d}\right)\right) y+D\right\}\right) \\
& \geq \mu\left(\left\{x: M_{n}(x) \leq D\right\}\right)=1 .
\end{aligned}
$$

So we have, in this case, an EVL for $M_{n}$ of type $E V_{3}$.

Proof of Theorem Q. We assume that by hypothesis for every $y \in \mathbb{R}$ and some sequence $u_{n}=u_{n}(y)$ such that $n \mu\left(\left\{x: \varphi(x)>u_{n}(y)\right\}\right) \underset{n \rightarrow \infty}{\longrightarrow} \tau(y)$, we have

$$
\lim _{n \rightarrow \infty} \mu\left(\left\{x: M_{n}(x) \leq u_{n}(y)\right\}\right)=H(\tau(y)) .
$$

Given $t>0$ and a sequence $\left\{\delta_{n}\right\}_{n \in \mathbb{N}} \subset \mathbb{R}^{+}$with $\delta_{n} \underset{n \rightarrow \infty}{\longrightarrow} 0$, we take $y \in \mathbb{R}$ such that $t=\tau(y)$ and define $\ell_{n}:=\left\lfloor t /\left(\kappa \rho(\zeta) \delta_{n}^{d}\right)\right\rfloor$. We can always find such $y$ because (1.6) is equivalent to (1.4) and $\varphi$ is of the form (1.9), where $g$ is of type $g_{i}$, for some $i \in\{1,2,3\}$, which implies that $\hat{M}_{n}$ has a limit law of type $E V_{i}$.

First we show that

$$
g^{-1}\left(u_{\ell_{n}}\right) \sim \delta_{n}
$$

If $n$ is sufficiently large, then

$$
\left\{x: \varphi(x)>u_{n}\right\}=\left\{x: g(\operatorname{dist}(x, \zeta))>u_{n}\right\}=\left\{x: \operatorname{dist}(x, \zeta)<g^{-1}\left(u_{n}\right)\right\}=B_{g^{-1}\left(u_{n}\right)}(\zeta) .
$$

Hence, by assumption on the sequence $u_{n}$, we have $n \mu\left(B_{g^{-1}\left(u_{n}\right)}(\zeta)\right) \underset{n \rightarrow \infty}{\longrightarrow} \tau(y)=t$. As Lebesgue's Differentiation Theorem holds for $\zeta \in \mathcal{X}$, we have $\frac{\mu\left(B_{\delta}(\zeta)\right)}{\left|B_{\delta}(\zeta)\right|} \rightarrow \rho(\zeta)$ as $\delta \rightarrow 0$. Consequently, since it is obvious that $g^{-1}\left(u_{n}\right) \rightarrow 0$ as $n \rightarrow \infty$, then $n\left|B_{g^{-1}\left(u_{n}\right)}(\zeta)\right| \underset{n \rightarrow \infty}{\longrightarrow}$ $t / \rho(\zeta)$. Thus, we may write $g^{-1}\left(u_{n}\right) \sim\left(\frac{t}{\kappa n \rho(\zeta)}\right)^{1 / d}$ and substituting $n$ by $\ell_{n}$ we are immediately led to (2.6) by definition of $\ell_{n}$.

Next, using Lebesgue's Differentiation Theorem, again, we get $\mu\left(B_{\delta_{n}}(\zeta)\right) \sim \rho(\zeta) \kappa \delta_{n}^{d}$ which easily implies that by definition of $\ell_{n}$,

$$
\frac{t}{\mu\left(B_{\delta_{n}}(\zeta)\right)} \sim \ell_{n}
$$

Now we note that, as in (2.1)

$$
\begin{aligned}
& \left\{x: M_{\ell_{n}}(x) \leq u_{\ell_{n}}\right\}=\bigcap_{j=0}^{\ell_{n}-1}\left\{x: X_{j}(x) \leq u_{\ell_{n}}\right\}=\bigcap_{j=0}^{\ell_{n}-1}\left\{x: g\left(\operatorname{dist}\left(f^{j}(x), \zeta\right)\right) \leq u_{\ell_{n}}\right\} \\
& =\bigcap_{j=0}^{\ell_{n}-1}\left\{x: \operatorname{dist}\left(f^{j}(x), \zeta\right) \geq g^{-1}\left(u_{\ell_{n}}\right)\right\}=\left\{x: r_{B_{g^{-1}\left(u_{\ell_{n}}\right)}(\zeta)}(x) \geq \ell_{n}\right\} .
\end{aligned}
$$


At this point, we claim that

$$
\lim _{n \rightarrow \infty} \mu\left(\left\{x: r_{B_{\delta_{n}}(\zeta)}(x) \geq \frac{t}{\mu\left(B_{\delta_{n}}(\zeta)\right)}\right\}\right)=\lim _{n \rightarrow \infty} \mu\left(\left\{x: M_{\ell_{n}}(x) \leq u_{\ell_{n}}\right\}\right) .
$$

Then, the first part of the theorem follows, once we observe that, by hypothesis, we have

$$
\mu\left(\left\{x: M_{\ell_{n}}(x) \leq u_{\ell_{n}}\right\}\right) \underset{n \rightarrow \infty}{\longrightarrow} H(\tau(y))=H(t) .
$$

The second part also follows since when the EVL of $M_{n}$ coincides with that of $\hat{M}_{n}$, then $H(\tau(y))=\mathrm{e}^{-\tau(y)}$. This is because in the i.i.d. setting (1.4) is equivalent to (1.6), as we have already mentioned.

It remains to show that (2.9) holds. First, observe that

$$
\begin{aligned}
& \mu\left(\left\{r_{B_{\delta_{n}}(\zeta)} \geq \frac{t}{\mu\left(B_{\delta_{n}}(\zeta)\right)}\right\}\right)=\mu\left(\left\{M_{\ell_{n}} \leq u_{\ell_{n}}\right\}\right)+\left(\mu\left(\left\{r_{B_{\delta_{n}}(\zeta)} \geq \ell_{n}\right\}\right)-\mu\left(\left\{M_{\ell_{n}} \leq u_{\ell_{n}}\right\}\right)\right) \\
& +\left(\mu\left(\left\{r_{B_{\delta_{n}}(\zeta)} \geq \frac{t}{\mu\left(B_{\delta_{n}}(\zeta)\right)}\right\}\right)-\mu\left(\left\{r_{B_{\delta_{n}}(\zeta)} \geq \ell_{n}\right\}\right)\right) .
\end{aligned}
$$

For the third term on the right, note that by (2.7) we have

$$
\begin{aligned}
\mid \mu\left(\left\{r_{B_{\delta_{n}}(\zeta)} \geq \ell_{n}\right\}\right)-\mu\left(\left\{r_{B_{\delta_{n}}(\zeta)}\right.\right. & \left.\left.\geq \frac{t}{\mu\left(B_{\delta_{n}}(\zeta)\right)}\right\}\right) \mid \\
& =\left|\mu\left(\left\{r_{B_{\delta_{n}}(\zeta)} \geq \ell_{n}\right\}\right)-\mu\left(\left\{r_{B_{\delta_{n}}(\zeta)} \geq\left(1+\varepsilon_{n}\right) \ell_{n}\right\}\right)\right|
\end{aligned}
$$

for some sequence $\left\{\varepsilon_{n}\right\}_{n} \in \mathbb{N}$ such that $\varepsilon_{n} \rightarrow 0$, as $n \rightarrow \infty$. By (2.5), (2.7) and stationarity it follows that

$$
\left|\mu\left(\left\{r_{B_{\delta_{n}}(\zeta)} \geq \ell_{n}\right\}\right)-\mu\left(\left\{r_{B_{\delta_{n}}(\zeta)} \geq\left(1+\varepsilon_{n}\right) \ell_{n}\right\}\right)\right| \leq\left|\varepsilon_{n}\right| \ell_{n} \mu\left(B_{\delta_{n}}(\zeta)\right) \sim\left|\varepsilon_{n}\right| t \rightarrow 0,
$$

as $n \rightarrow \infty$.

For the remaining term, using (2.6), (2.7) and (2.8), we have

$$
\begin{aligned}
& \left|\mu\left(\left\{r_{B_{\delta_{n}}(\zeta)} \geq \ell_{n}\right\}\right)-\mu\left(\left\{M_{\ell_{n}} \leq u_{\ell_{n}}\right\}\right)\right|=\left|\mu\left(\left\{r_{B_{\delta_{n}}(\zeta)} \geq \ell_{n}\right\}\right)-\mu\left(\left\{r_{B_{g^{-1}\left(u_{\ell_{n}}\right)}(\zeta)} \geq \ell_{n}\right\}\right)\right| \\
& \leq \sum_{i=1}^{\ell_{n}} \mu\left(f^{-i}\left(B_{\delta_{n}}(\zeta) \triangle B_{g^{-1}\left(u_{\ell_{n}}\right)}(\zeta)\right)\right) \\
& =\ell_{n} \mu\left(B_{\delta_{n}}(\zeta) \triangle B_{g^{-1}\left(u_{\ell_{n}}\right)}(\zeta)\right) \\
& \sim \frac{t}{\mu\left(B_{\delta_{n}}(\zeta)\right)}\left|\mu\left(B_{\delta_{n}}(\zeta)\right)-\mu\left(B_{g^{-1}\left(u_{\ell_{n}}\right)}(\zeta)\right)\right| \\
& =t\left|1-\frac{\mu\left(B_{g^{-1}\left(u_{\ell_{n}}\right)}(\zeta)\right)}{\mu\left(B_{\delta_{n}}(\zeta)\right)}\right| \rightarrow 0
\end{aligned}
$$

as $n \rightarrow \infty$, which ends the proof of (2.9) . 
Proof of Corollary 4. Let us assume the existence of HTS to balls around $\zeta$ (not necessarily exponential). Then the first part of Theorem 1 assures the existence of an EVL as in (1.3) for $M_{n}$ defined in (1.2). This fact and the hypothesis that $D_{1}\left(u_{n}\right)$ holds allows us to use [LLR, Theorem 3.7.1] to conclude that there is $\theta>0$ such that $\lim _{n \rightarrow \infty} \mu\left(M_{n} \leq u_{n}\right)=\mathrm{e}^{-\theta \tau}$. Finally, we use the first part of Theorem 2 to conclude that we have HTS to balls centred on $\zeta$ of exponential type.

\section{Relation Between hitting times And exceedance point processes}

We have already seen how to relate HTS and EVL. We next show that if we enrich the process and the statistics by considering either multiple returns or multiple exceedances we can take the parallelism even further.

Given a sequence $\left\{\delta_{n}\right\}_{n \in \mathbb{N}} \subset \mathbb{R}^{+}$such that $\delta_{n} \underset{n \rightarrow \infty}{\longrightarrow} 0$, for each $j \in \mathbb{N}$, we define the $j$-th waiting (or inter-hitting) time as

$$
w_{B_{\delta_{n}}(\zeta)}^{j}(x)=r_{B_{\delta_{n}}(\zeta)}\left(f^{w_{B_{\delta_{n}}(\zeta)}^{1}(x)+\cdots+w_{B_{\delta_{n}}(\zeta)}^{j-1}(x)}(x)\right),
$$

and the $j$-th hitting time as

$$
r_{B_{\delta_{n}}(\zeta)}^{j}(x)=\sum_{i=1}^{j} w_{B_{\delta_{n}}(\zeta)}^{i}(x) .
$$

We define the Hitting Times Point Process (HTPP) by counting the number of hitting times during the time interval $[0, t)$. However, since $\mu\left(B_{\delta_{n}}(\zeta)\right) \rightarrow 0$, as $n \rightarrow \infty$, then by Kac's Theorem, the expected waiting time between hits is diverging to $\infty$ as $n$ increases. This fact suggests a time re-scaling using the factor $v_{n}^{*}:=1 / \mu\left(B_{\delta_{n}}(\zeta)\right)$, which is precisely the expected inter-hitting time. Hence, for any $x \in \mathcal{X}$ and every $t \geq 0$ define

$$
N_{n}^{*}(t)=N_{n}^{*}([0, t), x):=\sup \left\{j: r_{B_{\delta_{n}}(\zeta)}^{j}(x) \leq v_{n}^{*} t\right\}=\sum_{j=0}^{\left\lfloor v_{n}^{*} t\right\rfloor} \mathbf{1}_{B_{\delta_{n}}(\zeta)} \circ f^{j}
$$

When $x \in B_{\delta_{n}}(\zeta)$ and we consider the conditional measure $\mu_{B_{\delta_{n}}(\zeta)}$ instead of $\mu$, then we refer to $N_{n}^{*}(t)$ as the Return Times Point Process (RTPP).

If we have exponential HTS, $\left(G(t)=\mathrm{e}^{-t}\right.$ in (1.13)), then the distribution of the waiting time before hitting $B_{\delta_{n}}(\zeta)$ is asymptotically exponential. Also, if we assume that our systems are mixing, because in that case we can think that the process gets renewed when we come back to $B_{\delta_{n}}(\zeta)$, then one may look at the hitting times as the sum of almost independent r.v.s that are almost exponentially distributed. Hence, one would expect that the hitting times, when properly re-scaled, should form a point process with a Poisson type behaviour at the limit.

As discussed in Section 1.2, for hyperbolic systems, it is indeed the case that we do get a Poisson Process as the limit of HTPP. The theory in [HSV, BSTV] and [BT] implies that 
if $f \in N F^{2}$ has an acip then we have a Poisson limit for the HTPP. We postpone a sketch of this fact to Section 5, in order to keep our focus on the relation between HTS and EVL here. However, we would like to remark that a key difference between proofs for the first hitting time and for showing that we have a Poisson Point Process, if we are using the theory started in [HSV], is that a further mixing condition is required.

Now, we turn to an EVL point of view. In this context, one is concerned with the occurrence of exceedances of the level $u_{n}$ for the stationary stochastic process $X_{0}, X_{1}, \ldots$ In particular, we are interested in counting the number of exceedances, among a random sample $X_{0}, \ldots, X_{n-1}$ of size $n$. As in the previous sections, we consider the stationary stochastic process defined by (1.1) and a sequence of levels $\left\{u_{n}\right\}_{n \in \mathbb{N}}$ such that $n \mu\left(X_{0}>u_{n}\right) \rightarrow \tau>0$, as $n \rightarrow \infty$. We define the exceedance point process (EPP) by counting the number of exceedances during the time interval $[0, t)$. We re-scale time using the factor $v_{n}:=1 / \mu\left(X>u_{n}\right)$ given by Kac's Theorem, again. Then for any $x \in \mathcal{X}$ and every $t \geq 0$, set

$$
N_{n}(t)=N_{n}([0, t), x):=\sum_{j=0}^{\left\lfloor v_{n} t\right\rfloor} \mathbf{1}_{X_{j}>u_{n}} .
$$

The limit laws for these point processes can be used to assess the impact and damage caused by rare events since they describe their time occurrences, their individual impacts and accumulated effects. Assuming that the process is mixing, we almost have a situation of many Bernoulli trials where the expected number of successes is almost constant $(n \mu(X>$ $\left.\left.u_{n}\right) \rightarrow \tau>0\right)$. Thus, we expect a Poisson law as a limit. In fact, one should expect that the exceedance instants, when properly normalised, should form a point process with a Poisson Process as a limit, also. This is the content of [LLR, Theorem 5.2.1] which states that under $D_{1}\left(u_{n}\right)$ and $D^{\prime}\left(u_{n}\right)$, the EPP $N_{n}$, when properly normalised, converges in distribution to a Poisson Process. (See [LLR, Chapter 5], [HHL] and references therein for more information on the subject).

Similarly to Theorems 1 and 2, we show that if there exists a limiting continuous time stochastic process for the HTPP, when properly normalised, then the same holds for the EPP and vice-versa. In the sequel $\stackrel{d}{\rightarrow}$ denotes convergence in distribution.

Theorem 3. Let $(\mathcal{X}, \mathcal{B}, \mu, f)$ be a dynamical system where $\mu$ is an acip and consider $\zeta \in \mathcal{X}$ for which Lebesgue's Differentiation Theorem holds. Suppose that for any sequence $\delta_{n} \underset{n \rightarrow \infty}{\longrightarrow} 0$ we have that the HTPP defined in (3.2) is such that $N_{n}^{*} \underset{n \rightarrow \infty}{\longrightarrow} N$, where $N$ is a continuous time stochastic process. Then, for the EPP defined in (3.3) we also have $N_{n} \underset{n \rightarrow \infty}{\stackrel{d}{\longrightarrow}} N$.

Proof. The result follows immediately once we set $\delta_{n}=g^{-1}\left(u_{n}\right)$ and observe that for every $j, n \in \mathbb{N}$ and $x \in \mathcal{X}$ we have $\left\{x: X_{j}>u_{n}\right\}=\left\{x: f^{j}(x) \in B_{g^{-1}\left(u_{n}\right)}(\zeta)\right\}$, which implies that $N_{n}(t)=N_{n}^{*}(t)$, for all $t \geq 0$. 
Corollary 5. Suppose that $f \in N F^{2}$ and $f$ has an acip $\mu$. Then, denoting by $N_{n}$ the associated EPP as in (3.3), we have $N_{n} \stackrel{d}{\rightarrow} N$, as $n \rightarrow \infty$, where $N$ denotes a Poisson Process with intensity 1.

The fact that the maps in this corollary satisfy the conditions of Theorem 3 follows from the sketch in Section 5. So the result is otherwise immediate.

Theorem 4. Let $(\mathcal{X}, \mathcal{B}, \mu, f)$ be a dynamical system where $\mu$ is an acip and consider $\zeta \in \mathcal{X}$ for which Lebesgue's Differentiation Theorem holds. Suppose that for a sequence of levels $\left\{u_{n}\right\}_{n \in \mathbb{N}}$ such that $n \mu\left(X_{0}>u_{n}\right) \rightarrow \tau>0$, as $n \rightarrow \infty$, the EPP defined in (3.3) is such

that $N_{n} \underset{n \rightarrow \infty}{\stackrel{d}{\longrightarrow}} N$, where $N$ is a continuous time stochastic process. Then, for the HTPP defined in (3.3) we also have $N_{n}^{*} \underset{n \rightarrow \infty}{\stackrel{d}{\longrightarrow}} N$.

Proof. Given a sequence $\left\{\delta_{n}\right\}_{n \in \mathbb{N}} \subset \mathbb{R}^{+}$with $\delta_{n} \underset{n \rightarrow \infty}{\longrightarrow} 0$ we define, as in the proof of Theorem 2, the sequence $\ell_{n}$ such that $\delta_{n} \sim g^{-1}\left(u_{\ell_{n}}\right)$. Set $k_{n}:=\max \left\{v_{n}^{*}, v_{\ell_{n}}\right\}$ and observe that $\left|N_{n}^{*}(t)-N_{\ell_{n}}(t)\right| \leq \sum_{j=0}^{k_{n}} \mathbf{1}_{B_{\delta_{n}}(\zeta) \triangle B_{g^{-1}\left(u_{\ell_{n}}\right)}(\zeta)} \circ f^{j}$. Using stationarity we get

$$
\begin{aligned}
\mu\left(\left|N_{n}^{*}(t)-N_{\ell_{n}}(t)\right|>0\right) & \leq k_{n} \mu\left(B_{\delta_{n}}(\zeta) \triangle B_{g^{-1}\left(u_{\ell_{n}}\right)}(\zeta)\right) \\
& =k_{n}\left|\mu\left(B_{\delta_{n}}(\zeta)\right)-\mu\left(B_{g^{-1}\left(u_{\ell_{n}}\right)}(\zeta)\right)\right| \underset{n \rightarrow \infty}{\longrightarrow} 0
\end{aligned}
$$

by definition of $\ell_{n}$. The result now follows immediately by Slutsky's Theorem (see [DM, Theorem 6.3.15]).

\section{Poisson Statistics via EVL}

As we have already mentioned, [LLR, Theorem 5.2.1] states that for a stationary stochastic process satisfying $D_{1}\left(u_{n}\right)$ and $D^{\prime}\left(u_{n}\right)$, the EPP $N_{n}$ defined in (3.3) converges in distribution to a Poisson Process.

The main result in [FF2 states that in order to prove an EVL for stationary stochastic processes arising from a dynamical system, it suffices to show conditions $D_{2}\left(u_{n}\right)$ and $D^{\prime}\left(u_{n}\right)$. This proved to be an advantage over [LLR, Theorem 3.5.2] since the mixing information of systems is usually known through decay of correlations that can be easily used to prove $D_{2}\left(u_{n}\right)$, as opposed to condition $D_{1}\left(u_{n}\right)$ appearing in [LLR, Theorem 3.5.2].

Our goal here is to prove that we still get the Poisson limit if we relax $D_{1}\left(u_{n}\right)$ so that it suffices to have sufficiently fast decay of correlations of the dynamical systems that generate the stochastic processes. However, for that purpose, one needs to strengthen $D_{2}\left(u_{n}\right)$ in order to cope with multiple events. (Something similar was necessary in the corresponding theory in [HSV].) For that reason we introduce condition $D_{3}\left(u_{n}\right)$ below, that still follows from sufficiently fast decay of correlations, as $D_{2}\left(u_{n}\right)$ did, and together with $D^{\prime}\left(u_{n}\right)$ allows us to obtain the Poisson limit for the EPP. 
Let $\mathcal{S}$ denote the semi-ring of subsets of $\mathbb{R}_{0}^{+}$whose elements are intervals of the type $[a, b)$, for $a, b \in \mathbb{R}_{0}^{+}$. Let $\mathcal{R}$ denote the ring generated by $\mathcal{S}$. Recall that for every $A \in \mathcal{R}$ there are $k \in \mathbb{N}$ and $k$ intervals $I_{1}, \ldots, I_{k} \in \mathcal{S}$ such that $A=\cup_{i=1}^{k} I_{j}$. In order to fix notation, let $a_{j}, b_{j} \in \mathbb{R}_{0}^{+}$be such that $I_{j}=\left[a_{j}, b_{j}\right) \in \mathcal{S}$. For $I=[a, b) \in \mathcal{S}$ and $\alpha \in \mathbb{R}$, we denote $\alpha I:=[\alpha a, \alpha b)$ and $I+\alpha:=[a+\alpha, b+\alpha)$. Similarly, for $A \in \mathcal{R}$ define $\alpha A:=\alpha I_{1} \cup \cdots \cup \alpha I_{k}$ and $A+\alpha:=\left(I_{1}+\alpha\right) \cup \cdots \cup\left(I_{k}+\alpha\right)$.

For every $A \in \mathcal{R}$ we define

$$
M(A):=\max \left\{X_{i}: i \in A \cap \mathbb{Z}\right\} .
$$

In the particular case where $A=[0, n)$ we simply write, as before, $M_{n}=M[0, n)$.

At this point, we propose:

Condition $\left(D_{3}\left(u_{n}\right)\right)$. Let $A \in \mathcal{R}$ and $t \in \mathbb{N}$. We say that $D_{3}\left(u_{n}\right)$ holds for the sequence $X_{0}, X_{1}, \ldots$ if

$$
\mu\left(\left\{X_{0}>u_{n}\right\} \cap\left\{M(A+t) \leq u_{n}\right\}\right)-\mu\left(\left\{X_{0}>u_{n}\right\}\right) \mu\left(\left\{M(A) \leq u_{n}\right\}\right) \leq \gamma(n, t),
$$

where $\gamma(n, t)$ is nonincreasing in $t$ for each $n$ and $n \gamma\left(n, t_{n}\right) \rightarrow 0$ as $n \rightarrow \infty$ for some sequence $t_{n}=o(n)$, which means that $t_{n} / n \rightarrow 0$ as $n \rightarrow \infty$.

Recalling the definition of the EPP $N_{n}(t)=N_{n}[0, t)$ given in (3.3), we set

$$
N_{n}[a, b):=N(b)-N(a)=\sum_{j=\left\lceil v_{n} a\right\rceil}^{\left\lfloor v_{n} b\right\rfloor} \mathbf{1}_{\left\{X_{j}>u_{n}\right\}} .
$$

We now state the main result of this section that gives the Poisson statistics for the EPP under $D_{3}\left(u_{n}\right)$ and $D^{\prime}\left(u_{n}\right)$.

Theorem 5. Let $X_{1}, X_{2}, \ldots$ be a stationary stochastic process for which conditions $D_{3}\left(u_{n}\right)$ and $D^{\prime}\left(u_{n}\right)$ hold for a sequence of levels $u_{n}$ such that $n \mu\left(X_{0}>u_{n}\right) \rightarrow \tau>0$, as $n \rightarrow \infty$. Then the EPP $N_{n}$ defined in (3.3) is such that $N_{n} \stackrel{d}{\rightarrow} N$, as $n \rightarrow \infty$, where $N$ denotes a Poisson Process with intensity 1.

As a consequence of this theorem, Theorem 4 and the results in [FF1] we get:

Corollary 6. For any Benedicks-Carleson quadratic map $f_{a}$ (with $a \in \mathcal{B C}$ ), consider a stochastic process $X_{0}, X_{1}, \ldots$ defined by (1.1) and (1.9), with $\zeta$ being either the critical point or the critical value. Then, denoting by $N_{n}$ the associated EPP as in (3.3), we have $N_{n} \stackrel{d}{\rightarrow} N$, as $n \rightarrow \infty$, where $N$ denotes a Poisson Process with intensity 1 . Moreover, if we consider $N_{n}^{*}$, the HTPP as in (3.2), for balls around either the critical point or the critical value, then the same limit also applies to $N_{n}^{*}$.

With minor adjustments to [Col2], we can use Theorem 5 to show that, similarly to Corollary 5, interval maps with exponential decay of correlations have Poisson statistics for the EPP. However, we will not state this result here, since we prove a more general result (which works in higher dimensions) in Section 6. 
4.1. Proofs of the results. In this section we prove Theorem 5 and Corollary 6 . The key is Proposition 1 whose proof we prepare with the following two Lemmas. These are very similar to ones in [FF2, Section 3] and [Col2, Section 3], but we redo them here for completeness and because, in contrast to the original ones, we need them to take care of events that depend on nonconsecutive random variables.

Lemma 4.1. For any $\ell \in \mathbb{N}$ and $u \in \mathbb{R}$ we have

$$
\sum_{j=0}^{\ell-1} \mu\left(X_{j}>u\right) \geq \mu\left(M_{\ell}>u\right) \geq \sum_{j=0}^{\ell-1} \mu\left(X_{j}>u\right)-\sum_{j=0}^{\ell-1} \sum_{i=0, i \neq j}^{\ell-1} \mu\left(\left\{X_{j}>u\right\} \cap\left\{X_{i}>u\right\}\right)
$$

Proof. This is a straightforward consequence of the formula for the probability of a multiple union of events. See for example the first Theorem of Chapter 4 in [Fe].

Lemma 4.2. Assume that $r, s, \ell, t$ are nonnegative integers. Suppose that $A, B \in \mathcal{R}$ are such that $A \subset B$. Set $\ell:=\#\{j \in \mathbb{N}: j \in B \backslash A\}$. Assume that $\min \{x: x \in A\} \geq r+t$ and let $A_{0}=[0, r+t)$. Then, we have

$$
0 \leq \mu(M(A) \leq u)-\mu(M(B) \leq u) \leq \ell \cdot \mu(X>u)
$$

and

$$
\begin{aligned}
\mid \mu\left(M\left(A_{0} \cup A\right) \leq u\right)-\mu(M(A) \leq u) & +\sum_{i=0}^{r-1} \mu(\{X>u\} \cap\{M(A-i) \leq u\}) \mid \\
& \leq 2 r \sum_{i=1}^{r-1} \mu\left(\{X>u\} \cap\left\{X_{i}>u\right\}\right)+t \mu(X>u) .
\end{aligned}
$$

Proof. By the law of total probability and stationarity we have, for any $i \geq 0$,

$$
\begin{aligned}
\mu(M(A) \leq u) & =\mu(M(B) \leq u)+\mu(\{M(A) \leq u\} \cap\{M(B \backslash A)>u\}) \\
& \leq \mu(M(B) \leq u)+\mu(M(B \backslash A)>u) \\
& \leq \mu(M(B) \leq u)+\ell \mu(X>u)
\end{aligned}
$$

and the first statement of the Lemma follows.

For the second statement observe that

$$
\left\{M\left(A_{0} \cup A\right) \leq u\right\}=\{M([0, r)) \leq u\} \cap\{M([r, r+t)) \leq u\} \cap\{M(A) \leq u\} .
$$

Consequently,

$$
(\{M([0, r)) \leq u\} \cap\{M(A) \leq u\}) \backslash\left\{M\left(A_{0} \cup A\right) \leq u\right\} \subset\{M([r, r+t))>u\} .
$$

Thus, using the first inequality of Lemma 4.1 we obtain

$$
\left|\mu(\{M([0, r)) \leq u\} \cap\{M(A) \leq u\})-\mu\left(\left\{M\left(A_{0} \cup A\right) \leq u\right\}\right)\right| \leq t \mu(X>u) .
$$


Using stationarity and the first inequality in Lemma 4.1 we have

$$
\begin{aligned}
\mu(\{M([0, r)) \leq u\} \cap\{M(A) \leq u\}) & =\mu(\{M(A) \leq u\})-\mu(\{M([0, r))>u\} \cap\{M(A) \leq u\}) \\
& \geq \mu(\{M(A) \leq u\})-\sum_{i=0}^{r-1} \mu\left(\left\{X_{i}>u\right\} \cap\{M(A) \leq u\}\right) .
\end{aligned}
$$

Now, by the second inequality in Lemma 4.1 we have

$$
\begin{aligned}
\mu(\{M([0, r)) \leq u\} \cap\{M(A) \leq u\}) \leq & \mu(\{M(A) \leq u\})-\sum_{i=0}^{r-1} \mu\left(\left\{X_{i}>u\right\} \cap\{M(A) \leq u\}\right) \\
& +\sum_{i=0}^{r-1} \sum_{\ell=0, i \neq \ell}^{r-1} \mu\left(\left\{X_{i}>u\right\} \cap\left\{X_{\ell}>u\right\} \cap\{M(A) \leq u\}\right) .
\end{aligned}
$$

Finally, stationarity and the last three inequalities give

$$
\begin{aligned}
\mid \mu(\{M([0, r)) \leq u\} \cap\{M(A) \leq u\})-\mu(\{M(A) \leq u\}) & +\sum_{i=0}^{r-1} \mu(\{X>u\} \cap\{M(A-i) \leq u\}) \mid \\
& \leq 2 r \sum_{i=1}^{r-1} \mu\left(\{X>u\} \cap\left\{X_{i}>u\right\}\right),
\end{aligned}
$$

and the result follows by (4.3).

Proposition 1. Let $A \in \mathcal{R}$ be such that that $A=\bigcup_{j=1}^{p} I_{j}$ where $I_{j}=\left[a_{j}, b_{j}\right) \in \mathcal{S}$, $j=1, \ldots, p$ and $a_{1}<b_{1}<a_{2}<\cdots<b_{p-1}<a_{p}<b_{p}$. Let $\left\{u_{n}\right\}_{n \in \mathbb{N}}$ be such that $n \mu\left(X_{0}>u_{n}\right) \rightarrow \tau>0$, as $n \rightarrow \infty$, for some $\tau \geq 0$. Assume that conditions $D_{3}\left(u_{n}\right)$ and $D^{\prime}\left(u_{n}\right)$ hold. Then,

$$
\mu\left(M(n A) \leq u_{n}\right) \underset{n \rightarrow+\infty}{\longrightarrow} \prod_{j=1}^{p} \mu\left(M\left(n I_{j}\right) \leq u_{n}\right)=\prod_{j=1}^{p} e^{-\tau\left(b_{j}-a_{j}\right)} .
$$

Proof. Let $h:=\inf _{j \in\{1, \ldots, p\}}\left\{b_{j}-a_{j}\right\}$ and $H:=\lceil\sup \{x: x \in A\}\rceil$. Take $k>2 / h$ and $n$ sufficiently large. Note this guarantees that if we partition $n[0, H] \cap \mathbb{Z}$ into blocks of length $r_{n}:=\lfloor n / k\rfloor, J_{1}=\left[H n-r_{n}, H n\right), J_{2}=\left[H n-2 r_{n}, H n-r_{n}\right), \ldots, J_{H k}=\left[H n-H k r_{n}, n-\right.$ $\left.(H k-1) r_{n}\right), J_{H k+1}=\left[0, H n-H k r_{n}\right)$, then there is more than one of these blocks contained in $n I_{i}$. Let $S_{\ell}=S_{\ell}(k)$ be the number of blocks $J_{j}$ contained in $n I_{\ell}$, that is,

$$
S_{\ell}:=\#\left\{j \in\{1, \ldots, H k\}: J_{j} \subset n I_{\ell}\right\} .
$$

As we have already observed $S_{\ell}>1 \forall \ell \in\{1, \ldots, p\}$. For each $\ell \in\{1, \ldots, p\}$, we define

$$
A_{\ell}:=\bigcup_{i=1}^{\ell} I_{p-i+1} \text {. }
$$


Set $i_{\ell}:=\min \left\{j \in\{1, \ldots, k\}: J_{j} \subset n I_{\ell}\right\}$. Then $J_{i_{\ell}}, J_{i_{\ell}+1}, \ldots, J_{i_{\ell}+s_{\ell}} \subset n I_{\ell}$. Now, fix $\ell$ and for each $i \in\left\{i_{p-\ell+1}, \ldots, i_{p-\ell+1}+S_{p-\ell+1}\right\}$ let

$$
B_{i}:=\bigcup_{j=i_{p-\ell+1}}^{i} J_{j}, J_{i}^{*}:=\left[H n-i r_{n}, H n-(i-1) r_{n}-t_{n}\right) \text { and } J_{i}^{\prime}:=J_{i}-J_{i}^{*} \text {. }
$$

Note that $\left|J_{i}^{*}\right|=r_{n}-t_{n}$ and $\left|J_{i}^{\prime}\right|=t_{n}$. See Figure 1 for more of an idea of the notation here.

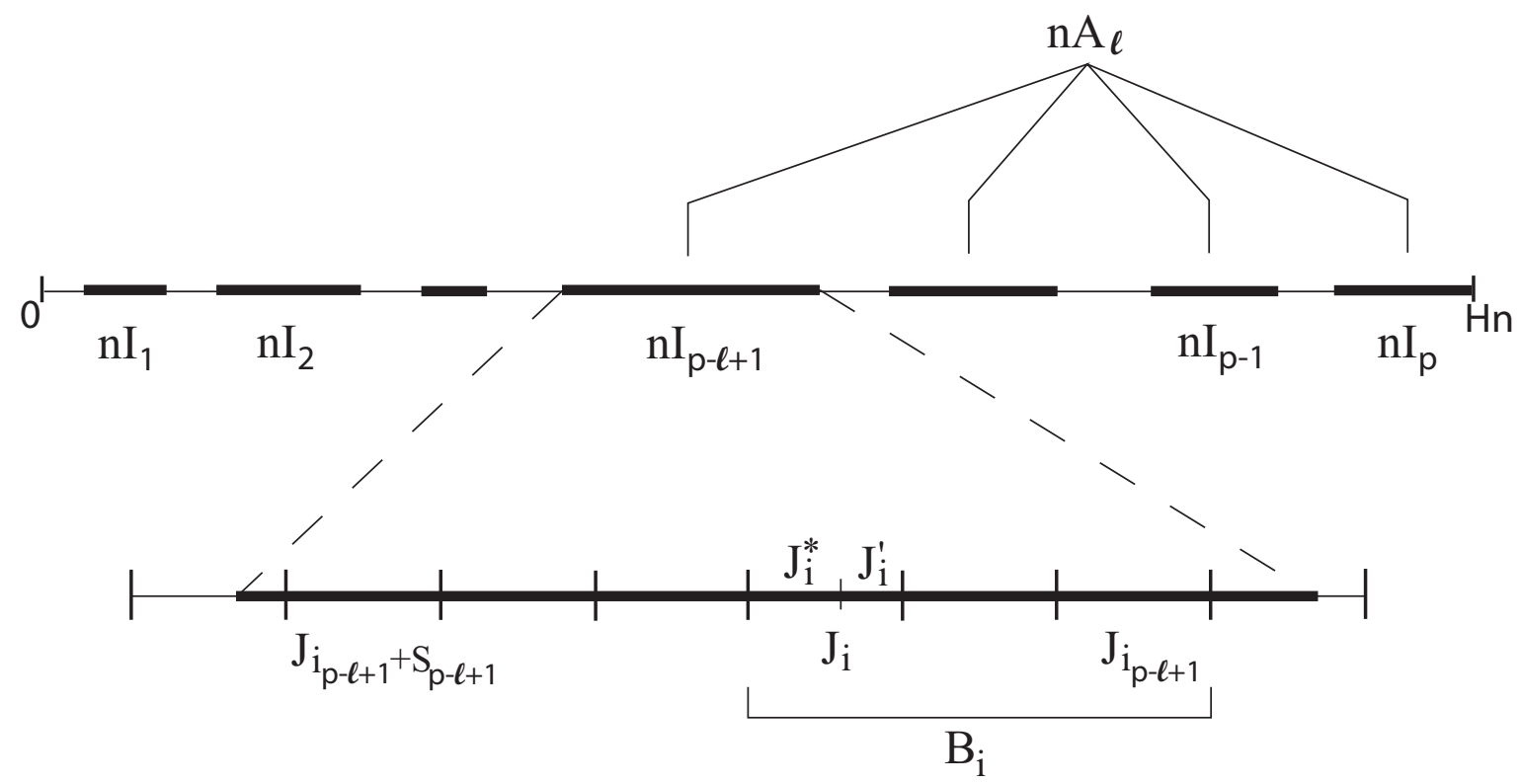

FiguRE 1. Notation 
We have,

$$
\begin{aligned}
\left|\mu\left(M\left(B_{i} \cup n A_{\ell-1}\right) \leq u_{n}\right)-\left(1-r_{n} \mu\left(X>u_{n}\right)\right) \mu\left(M\left(B_{i-1} \cup n A_{\ell-1}\right) \leq u_{n}\right)\right| & \\
= & \mid \mu\left(M\left(B_{i} \cup n A_{\ell-1}\right) \leq u_{n}\right)-\mu\left(M\left(B_{i-1} \cup n A_{\ell-1}\right) \leq u_{n}\right) \\
& +r_{n} \mu\left(X>u_{n}\right) \mu\left(M\left(B_{i-1} \cup n A_{\ell-1}\right) \leq u_{n}\right) \mid \\
\leq & \mid \mu\left(M\left(B_{i} \cup n A_{\ell-1}\right) \leq u_{n}\right)-\mu\left(M\left(B_{i-1} \cup n A_{\ell-1}\right) \leq u_{n}\right) \\
& \quad+\left(r_{n}-t_{n}\right) \mu\left(X>u_{n}\right) \mu\left(M\left(B_{i-1} \cup n A_{\ell-1}\right) \leq u_{n}\right) \mid \\
& +t_{n} \mu\left(X>u_{n}\right) \mu\left(M\left(B_{i-1} \cup n A_{\ell-1}\right) \leq u_{n}\right) \\
\leq & \mid \mu\left(M\left(B_{i} \cup n A_{\ell-1}\right) \leq u_{n}\right)-\mu\left(M\left(B_{i-1} \cup n A_{\ell-1}\right) \leq u_{n}\right) \\
& \quad+\sum_{j=0}^{r_{n}-t_{n}-1} \mu\left(\left\{X_{j+H n-i r_{n}}>u_{n}\right\} \cap\left\{M\left(B_{i} \cup n A_{\ell-1}\right) \leq u_{n}\right)\right\} \mid \\
& +\mid\left(r_{n}-t_{n}\right) \mu\left(X>u_{n}\right) \mu\left(M\left(B_{i-1} \cup n A_{\ell-1}\right) \leq u_{n}\right) \\
& \quad-\sum_{j=0}^{r_{n}-t_{n}-1} \mu\left(\left\{X_{j+H n-i r_{n}}>u_{n}\right\} \cap\left\{M\left(B_{i} \cup n A_{\ell-1}\right) \leq u_{n}\right)\right\} \mid \\
& +t_{n} \mu\left(X>u_{n}\right) .
\end{aligned}
$$

By Lemma 4.2, we obtain

$$
\begin{gathered}
\left|\mu\left(M\left(B_{i} \cup n A_{\ell-1}\right) \leq u_{n}\right)-\left(1-r_{n} \mu\left(X>u_{n}\right)\right) \mu\left(M\left(B_{i-1} \cup n A_{\ell-1}\right) \leq u_{n}\right)\right| \\
\leq 2\left(r_{n}-t_{n}\right) \sum_{j=1}^{r_{n}-t_{n}-1} \mu\left(\left\{X>u_{n}\right\} \cap\left\{X_{j}>u_{n}\right\}\right)+t_{n} \mu\left(X>u_{n}\right) \\
\quad+\sum_{j=0}^{r_{n}-t_{n}-1} \mid \mu\left(X>u_{n}\right) \mu\left(M\left(B_{i-1} \cup n A_{\ell-1}\right) \leq u_{n}\right) \\
\quad-\mu\left(\left\{X>u_{n}\right\} \cap\left\{M\left(\left(B_{i-1} \cup n A_{\ell-1}\right)-d_{j}\right) \leq u_{n}\right\}\right) \mid \\
+t_{n} \mu\left(X>u_{n}\right),
\end{gathered}
$$

where $d_{j}=\left(j+H n-i r_{n}\right)$. Now using condition $D_{3}\left(u_{n}\right)$, we obtain

$$
\begin{aligned}
& \left|\mu\left(M\left(B_{i} \cup n A_{\ell-1}\right) \leq u_{n}\right)-\left(1-r_{n} \mu\left(X>u_{n}\right)\right) \mu\left(M\left(B_{i-1} \cup n A_{\ell-1}\right) \leq u_{n}\right)\right| \\
& \quad \leq 2\left(r_{n}-t_{n}\right) \sum_{j=1}^{r_{n}-t_{n}-1} \mu\left(\left\{X>u_{n}\right\} \cap\left\{X_{j}>u_{n}\right\}\right)+2 t_{n} \mu\left(X>u_{n}\right)+\left(r_{n}-t_{n}\right) \gamma\left(n, t_{n}\right) .
\end{aligned}
$$


Set

$$
\Upsilon_{k, n}:=2\left(r_{n}-t_{n}\right) \sum_{j=1}^{r_{n}-t_{n}-1} \mu\left(\left\{X>u_{n}\right\} \cap\left\{X_{j}>u_{n}\right\}\right)+2 t_{n} \mu\left(X>u_{n}\right)+\left(r_{n}-t_{n}\right) \gamma\left(n, t_{n}\right) .
$$

Recalling (1.4), we may assume that $n$ and $k$ are sufficiently large so that $\frac{n}{k} \mu\left(X>u_{n}\right)<2$ and $\left|1-r_{n} \mu\left(X>u_{n}\right)\right|<1$ which implies

$\left|\mu\left(M\left(B_{S_{p-\ell+1}} \cup n A_{\ell-1}\right) \leq u_{n}\right)-\left(1-r_{n} \mu\left(X>u_{n}\right)\right) \mu\left(M\left(B_{S_{p-\ell+1}-1} \cup n A_{\ell-1}\right) \leq u_{n}\right)\right| \leq \Upsilon_{k, n}$,

and

$$
\begin{aligned}
& \left|\mu\left(M\left(B_{S_{p-\ell+1}} \cup n A_{\ell-1}\right) \leq u_{n}\right)-\left(1-r_{n} \mu\left(X>u_{n}\right)\right)^{2} \mu\left(M\left(B_{S_{p-\ell+1}-2} \cup n A_{\ell-1}\right) \leq u_{n}\right)\right| \\
& \leq\left|\mu\left(M\left(B_{S_{p-\ell+1}} \cup n A_{\ell-1}\right) \leq u_{n}\right)-\left(1-r_{n} \mu\left(X>u_{n}\right)\right) \mu\left(M\left(B_{S_{p-\ell+1}-1} \cup n A_{\ell-1}\right) \leq u_{n}\right)\right| \\
& +\left|1-r_{n} \mu\left(X>u_{n}\right)\right| \mid \mu\left(M\left(B_{S_{p-\ell+1}-1} \cup n A_{\ell-1}\right) \leq u_{n}\right) \\
& -\left(1-r_{n} \mu\left(X>u_{n}\right)\right) \mu\left(M\left(B_{S_{p-\ell+1}-2} \cup n A_{\ell-1}\right) \mid\right.
\end{aligned}
$$

$\leq 2 \Upsilon_{k, n}$

Inductively, we obtain

$\left|\mu\left(M\left(B_{S_{p-l+1}} \cup n A_{\ell-1}\right) \leq u_{n}\right)-\left(1-r_{n} \mu\left(X>u_{n}\right)\right)^{S_{p-\ell+1}} \mu\left(M\left(n A_{\ell-1}\right) \leq u_{n}\right)\right| \leq S_{p-\ell+1} \Upsilon_{k, n}$.

Using Lemma 4.2,

$$
\begin{aligned}
& \quad\left|\mu\left(M\left(n A_{\ell}\right) \leq u_{n}\right)-\left(1-r_{n} \mu\left(X>u_{n}\right)\right)^{S_{p-\ell+1}} \mu\left(M\left(n A_{\ell-1}\right) \leq u_{n}\right)\right| \\
& \quad \leq\left|\mu\left(M\left(n A_{\ell}\right) \leq u_{n}\right)-\mu\left(M\left(B_{S_{p-l+1}} \cup n A_{\ell-1}\right) \leq u_{n}\right)\right| \\
& \quad \quad+\left|\mu\left(M\left(B_{S_{p-l+1}} \cup n A_{\ell-1}\right) \leq u_{n}\right)-\left(1-r_{n} \mu\left(X>u_{n}\right)\right)^{S_{p-\ell+1}} \mu\left(M\left(n A_{\ell-1}\right) \leq u_{n}\right)\right| \\
& \quad \leq\left|\mu\left(M\left(n I_{p-\ell+1} \cup n A_{\ell-1}\right) \leq u_{n}\right)-\mu\left(M\left(\cup_{i=i_{\ell}}^{S_{p-\ell+1}} J_{i} \cup n A_{\ell-1}\right) \leq u_{n}\right)\right|+S_{p-l+1} \Upsilon_{k, n} \\
& \leq 2 r_{n} \mu\left(X>u_{n}\right)+S_{p-l+1} \Upsilon_{k, n} .
\end{aligned}
$$

In the next step we have

$$
\begin{aligned}
& \left|\mu\left(M\left(n A_{\ell}\right) \leq u_{n}\right)-\left(1-r_{n} \mu\left(X>u_{n}\right)\right)^{S_{p-\ell+1}+S_{p-\ell+2}} \mu\left(M\left(n A_{\ell-2}\right) \leq u_{n}\right)\right| \\
& \leq\left|\mu\left(M\left(n A_{\ell}\right) \leq u_{n}\right)-\left(1-r_{n} \mu\left(X>u_{n}\right)\right)^{S_{p-\ell+1}} \mu\left(M\left(n A_{\ell-1}\right) \leq u_{n}\right)\right| \\
& \quad+\left|1-r_{n} \mu\left(X>u_{n}\right)\right|^{S_{p-\ell+1}} \mid \mu\left(M\left(n A_{\ell-1}\right) \leq u_{n}\right) \\
& \quad-\left(1-r_{n} \mu\left(X>u_{n}\right)\right)^{S_{p-\ell+2}} \mu\left(M\left(n A_{\ell-2}\right) \leq u_{n}\right) \mid \\
& \leq 4 r_{n} \mu\left(X>u_{n}\right)+\left(S_{p-\ell+1}+S_{p-\ell+2}\right) \Upsilon_{k, n} .
\end{aligned}
$$


Therefore, by induction, we obtain

$$
\left|\mu\left(M\left(n A_{p}\right) \leq u_{n}\right)-\left(1-r_{n} \mu\left(X>u_{n}\right)\right)^{\sum_{j=1}^{p} S_{j}}\right| \leq 2 p r_{n} \mu\left(X>u_{n}\right)+\sum_{j=1}^{p} S_{j} \Upsilon_{k, n}
$$

Now, it is easy to see that $S_{j} \sim k\left|I_{j}\right|$, for each $j \in\{1, \ldots, p\}$. Consequently,

$$
\begin{aligned}
& \lim _{k \rightarrow+\infty} \lim _{n \rightarrow+\infty}\left(1-r_{n} \mu\left(X>u_{n}\right)\right)^{\sum_{j=1}^{p} S_{j}}=\lim _{k \rightarrow+\infty} \lim _{n \rightarrow+\infty}\left(1-\left\lfloor\frac{n}{k}\right\rfloor \mu\left(X>u_{n}\right)\right)^{\sum_{j=1}^{p} S_{j}}
\end{aligned}
$$

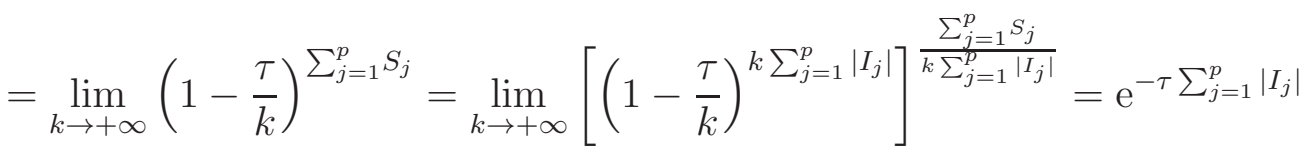

$$
\begin{aligned}
& =\prod_{j=1}^{p} \mathrm{e}^{-\tau\left(b_{j}-a_{j}\right)} \text {. }
\end{aligned}
$$

To conclude the proof it suffices to show that

$$
\lim _{k \rightarrow+\infty} \lim _{n \rightarrow+\infty}\left(2 p r_{n} \mu\left(X>u_{n}\right)+k H \Upsilon_{k, n}\right)=0 .
$$

We start by noting that, since $n \mu\left(X>u_{n}\right) \rightarrow \tau \geq 0$,

$$
\lim _{k \rightarrow+\infty} \lim _{n \rightarrow+\infty} 2 p r_{n} \mu\left(X>u_{n}\right)=\lim _{k \rightarrow+\infty} \frac{2 p \tau}{k}=0 .
$$

Next we need to check that

$$
\lim _{k \rightarrow+\infty} \lim _{n \rightarrow+\infty} k \Upsilon_{k, n}=0
$$

which means,

$$
\begin{array}{r}
\lim _{k \rightarrow+\infty} \lim _{n \rightarrow+\infty} 2 k\left(r_{n}-t_{n}\right) \sum_{j=1}^{r_{n}-t_{n}-1} \mu\left(\left\{X>u_{n}\right\} \cap\left\{X_{j}>u_{n}\right\}\right)+2 k t_{n} \mu\left(X>u_{n}\right) \\
+k\left(r_{n}-t_{n}\right) \gamma\left(n, t_{n}\right)=0 .
\end{array}
$$

Assume that $t=t_{n}$ where $t_{n}=o(n)$ is given by Condition $D_{3}\left(u_{n}\right)$. Now, observe that, by (1.4), for every $k \in \mathbb{N}$, we have $\lim _{n \rightarrow \infty} k t_{n} \mu\left(X>u_{n}\right)=0$. Finally, use $D_{3}\left(u_{n}\right)$ and $D^{\prime}\left(u_{n}\right)$ to prove that the two remaining terms also go to 0 .

Proof of Theorem 5. Since the Poisson Process has no fixed atoms, that is, points $t$ such that $\mu(N(\{t\})>0)>0$, the convergence is equivalent to convergence of finite dimensional distributions. But, because $N$ is a simple point process, without multiple events, we may use a criterion proposed by Kallenberg [Ka, Theorem 4.7] to show the stated convergence. Namely we need to verify that

(1) $\mathbb{E}\left(N_{n}(I)\right) \underset{n \rightarrow \infty}{\longrightarrow} \mathbb{E}(N(I))$, for all $I \in \mathcal{S}$;

(2) $\mu\left(N_{n}(A)=0\right) \underset{n \rightarrow \infty}{\longrightarrow} \mu(N(A)=0)$, for all $A \in \mathcal{R}$, 
where $\mathbb{E}(\cdot)$ denotes the expectation with respect to $\mu$.

First we show that condition (1) holds. Let $a, b \in \mathbb{R}^{+}$be such that $I=[a, b)$, then, recalling that $v_{n}=1 / \mu\left(X_{0}>u_{n}\right)$, we have

$$
\begin{aligned}
\mathbb{E}\left(N_{n}(I)\right) & =\mathbb{E}\left(\sum_{j=\left\lfloor v_{n} a\right\rfloor+1}^{\left\lfloor v_{n} b\right\rfloor} \mathbf{1}_{\left\{X_{j}>u_{n}\right\}}\right)=\sum_{j=\left\lfloor v_{n} a\right\rfloor+1}^{\left\lfloor v_{n} b\right\rfloor} E\left(\mathbf{1}_{\left\{X_{j}>u_{n}\right\}}\right) \\
& =\left(\left\lfloor v_{n} b\right\rfloor-\left(\left\lfloor v_{n} a\right\rfloor+1\right)\right) \mu\left(X_{0}>u_{n}\right) \\
& \sim(b-a) v_{n} \mu\left(X_{0}>u_{n}\right) \underset{n \rightarrow \infty}{\longrightarrow}(b-a)=\mathbb{E}(N(I)) .
\end{aligned}
$$

To prove condition (2), let $s \in \mathbb{N}$ and $A=\cup_{i=1}^{s} I_{i}$ where $I_{1}, \ldots, I_{s} \in \mathcal{S}$ are disjoint. Also let $a_{j}, b_{j} \in \mathbb{R}^{+}$be such that $I_{j}=\left[a_{j}, b_{j}\right)$. By Proposition 1 , we have

$\mu\left(N_{n}(A)=0\right)=\mu\left(\cap_{i=1}^{s}\left\{M\left(v_{n} I_{j}\right) \leq u_{n}\right\}\right) \sim \mu\left(\cap_{i=1}^{s}\left\{M\left((n / \tau) I_{j}\right) \leq u_{n}\right\}\right) \underset{n \rightarrow \infty}{\longrightarrow} \prod_{j=1}^{s} \mathrm{e}^{-\left(b_{j}-a_{j}\right)}$.

The result follows at once since $\mu(N(A)=0)=\prod_{i=1}^{s} \mu\left(N\left(I_{j}\right)=0\right)=\prod_{j=1}^{s} \mathrm{e}^{-\left(b_{j}-a_{j}\right)}$.

Proof of Corollary [6. In [FF1, FF2], conditions $D_{2}\left(u_{n}\right)$ and $D^{\prime}\left(u_{n}\right)$ were proved for stochastic processes $X_{0}, X_{1}, \ldots$ as in (1.1) and (1.9) with $\zeta$ being either the critical point or the critical value and observables of type $g_{3}\left(g_{3}(x)=x\right.$ for $\zeta=1$ and $g_{3}=1-a x^{2}$ for $\zeta=0)$.

Observe that independently of the type of $g$, the sequence $u_{n}$ is computed so that an exceedance of the level $u_{n}$ corresponds to a visit to the ball $B_{\delta_{n}}(\zeta)$, where $\delta_{n}$ is such that $\mu\left(B_{\delta_{n}}\right) \sim \tau / n$. This means that condition $D^{\prime}\left(u_{n}\right)$ can be written in terms of returns to $B_{\delta_{n}}(\zeta)$ which implies that it holds for every sequence $X_{0}, X_{1}, \ldots$, independently of the shape of $g$.

Condition $D_{3}\left(u_{n}\right)$ follows from decay of correlations. In fact, from [KN, Y1] one has that for all $\phi, \psi: M \rightarrow \mathbb{R}$ with bounded variation, there is $C, \alpha>0$ independent of $\phi, \psi$ and $n$ such that

$$
\left|\int \phi \cdot\left(\psi \circ f^{t}\right) d \mu-\int \phi d \mu \int \psi d \mu\right| \leq C \operatorname{Var}(\phi)\|\psi\|_{\infty} \mathrm{e}^{-\alpha t}, \quad \forall t \in \mathbb{N}_{0},
$$

where $\operatorname{Var}(\phi)$ denotes the total variation of $\phi$. For each $A \in \mathcal{R}$, take $\phi=\mathbf{1}_{\left\{X_{0}>u_{n}\right\}}$ and $\psi=$ $\mathbf{1}_{\left\{M(A) \leq u_{n}\right\}}$, then (4.4) implies that Condition $D_{3}\left(u_{n}\right)$ holds with $\gamma(n, t)=\gamma(t):=2 C \mathrm{e}^{-\alpha t}$ and for the sequence $t_{n}=\sqrt{n}$, for example.

\section{Poisson Statistics for first Return times}

The purpose of this section is to discuss what is known about the Poisson statistics of first return times to balls. The main focus is on showing that a map $f \in N F^{2}$ with an acip must have the RTPP asymptotically converging to a Poisson Process. However, for more 
generality we will introduce the ideas assuming that our phase space $\mathcal{X}$ is a Riemannian manifold. We note that a similar result to the main theorem [HLV implies that the limit laws for the HTPP and RTPP are the same. So since the results we will cite below are usually given in terms of RTS, we will use this.

Similarly to the proof of Theorem 5 , in order to show that the RTPP has a Poisson limit, it suffices to prove that for $k \in \mathbb{N}$ and a rectangle $R_{k} \subset \mathbb{R}^{k}$,

$$
\left|\mu_{U_{n}}\left(\left(w_{U_{n}}, w_{U_{n}}^{2}, \ldots, w_{U_{n}}^{k}\right) \in \frac{1}{\mu\left(U_{n}\right)} R_{k}\right)-\int_{R_{k}} \Pi_{i=1}^{k} \mathrm{e}^{t_{i}} d t^{k}\right| \rightarrow 0 \text { as } n \rightarrow \infty .
$$

The main result of [BSTV] is that the RTS for an inducing scheme is the same for the inducing scheme as for the original system. However, they remark in that paper that their methods extend to give the same Poisson statistics for the inducing scheme and the original system. In [BT], the theory in [BSTV] was extended to show that for multimodal maps of the interval the RTS of suitable inducing schemes converge to the RTS of the original system. The corresponding result for Poisson statistics follows similarly. For multimodal maps $f: I \rightarrow I$, with an acip $\mu$, those inducing schemes are Rychlik maps. Therefore to prove that the original $(I, f, \mu)$ has the RTPP converging to a Poisson process, we must show that the induced, Rychlik, maps also have this property. As we sketch below, this can be proved using [HSV, Theorem 2.6].

For a system $(X, F, \mu)$, we say that a partition $\mathcal{Q}$ is uniform mixing if there exists $\gamma_{\mathcal{Q}}(n) \rightarrow 0$ as $n \rightarrow \infty$, such that

$$
\gamma_{\mathcal{Q}}(n):=\sup _{k, l} \sup _{\substack{A \in \sigma \mathcal{Q}_{k} \\ B \in F^{-(n+k)} \sigma \mathcal{Q}_{l}}}|\mu(A \cap B)-\mu(A) \mu(B)| .
$$

Here $\mathcal{Q}_{k}:=\bigvee_{j=0}^{k-1} F^{-j} \mathcal{Q}$ and $\sigma \mathcal{Q}_{k}$ is the sigma algebra generated by $\mathcal{Q}_{k}$. For our purposes $\mathcal{Q}$ will be $\left\{U, U^{c}\right\}$ where $U$ is a ball around $\zeta$.

By [HSV, Theorem 2.6], if we assume the system is uniform mixing for $\left\{U, U^{c}\right\}$, then for a rectangle $R_{k} \subset \mathbb{R}^{k}$,

$$
\left|\mu_{U}\left(\left(w_{U}, w_{U}^{2}, \ldots, w_{U}^{k}\right) \in \frac{1}{\mu(U)} R_{k}\right)-\int_{R_{k}} \Pi_{i=1}^{k} \mathrm{e}^{t_{i}} d t^{k}\right| \leq \operatorname{Err}(k, U) .
$$

Moreover, the term $\operatorname{Err}(k, U)$ goes to 0 as $U$ shrinks to a point $\zeta$. In fact, we have $\operatorname{Err}(k, U)=k(3 d(U)+R(k, U))$ where $R(k, U) \rightarrow 0$ as $\mu(U) \rightarrow 0$ and the rate that $R(k, U)$ goes to zero depends on how $\gamma_{\mathcal{Q}}$ shrinks with $U$. As was shown in [BSTV], for Rychlik maps the quantity $d(U)$ tends 0 as $U \rightarrow\{\zeta\}$. Therefore it only remains to show that the Rychlik maps defined in [BT] are uniform mixing for $\left\{U, U^{c}\right\}$.

Since we assumed that $(X, F, \mu)$ is Rychlik, [R, Theorem 5] implies that the natural partition $\mathcal{P}_{1}$, consisting of maximal intervals on which $f$ is a homeomorphism, is Bernoulli, with exponential speed. Since $(X, F, \mu)$ is uniformly expanding, this implies that $\left\{U, U^{c}\right\}$ is also Bernoulli, with exponential speed. As noted in [HSV, Remark 2.5], this implies that $\left\{U, U^{c}\right\}$ is uniform mixing, as required. 
The proof that the successive returns form a point process converging to a Poisson Process follows from (5.1) and the Kallenberg argument used in the proof of Theorem 5.

\section{EVL AND HTS IN HIGHER DIMENSIONS}

In this section, we extend Collet's theory of maps with exponential decay of correlations from one dimension to higher dimensions. We conclude with an example.

Let $\mathcal{X}$ be as usual a $d$-dimensional compact Riemannian manifold and $f: \mathcal{X} \rightarrow \mathcal{X}$ a $C^{2}$ endomorphism. We say that $f$ admits a Young tower if there exists a ball $\Delta \subset \mathcal{X}$, a countable partition $\mathcal{P}(\bmod 0)$ of $\Delta$ into topological balls $\Delta_{i}$ with smooth boundaries, and a return time function $R: \Delta \rightarrow \mathbb{N}$ piecewise constant on elements of $\mathcal{P}$ satisfying the following properties:

$\left(\mathbf{Y}_{1}\right)$ Markov: for each $\Delta_{i} \in \mathcal{P}$ and $R=R\left(\Delta_{i}\right), f^{R}: \Delta_{i} \rightarrow \Delta$ is a $C^{2}$ diffeomorphism (and in particular a bijection). Thus the induced map

$$
F: \Delta \rightarrow \Delta \text { given by } F(x)=f^{R(x)}(x)
$$

is defined almost everywhere and satisfies the classical Markov property. We consider also the separation time $s(x, y)$ given by the maximum integer such that $F^{i}(x)$ and $F^{i}(y)$ belong to the same element of the partition $\mathcal{P}$ for all $i \leq s(x, y)$, which we assume to be defined and finite for almost every pair of points $x, y \in \Delta$.

$\left(\mathbf{Y}_{2}\right)$ Uniform backward contraction: There exist $C>0$ and $0<\beta<1$ such that for $x, y \in \Delta$ and any $0 \leq n \leq s(x, y)$ we have

$$
\operatorname{dist}\left(f^{n}(x), f^{n}(y)\right) \leq C \beta^{s(x, y)-n} .
$$

$\left(\mathbf{Y}_{3}\right)$ Bounded distortion: For any $x, y \in \Delta$ and any $0 \leq k \leq n<s(x, y)$ we have

$$
\log \prod_{i=k}^{n} \frac{\operatorname{det} D f\left(f^{i}(x)\right)}{\operatorname{det} D f\left(f^{i}(x)\right)} \leq C \beta^{s(x, y)-n}
$$

$\left(\mathbf{Y}_{4}\right)$ Integrable return times:

$$
\int R d \text { Leb }<\infty
$$

In this section we only consider maps admitting a Young tower with exponential return time tail which means that we will replace condition $\left(\mathbf{Y}_{4}\right)$ by the following stronger one

$\left(\mathbf{Y}_{4}^{\prime}\right)$ Exponential tail decay: There is $C, \alpha>0$ such that

$$
\operatorname{Leb}(\{R>n\})=C \mathrm{e}^{-\alpha n} \text {. }
$$

These systems have been studied, in a more general context, by L.S. Young in [Y2, Y3], where several examples can also be found. Among the properties proved by L.S. Young we mention the existence of an $F$-invariant measure $\mu_{0}$ that is equivalent to Lebesgue measure on $\Delta$ (meaning that its density is bounded above and below by a constant). After 
saturating one gets an absolutely continuous (w.r.t. Lebesgue), $f$-invariant probability given by

$$
\mu(A)=\bar{R}^{-1} \sum_{\ell=0}^{\infty} \mu_{0}\left(f^{-\ell}(A) \cap\{R>\ell\}\right),
$$

where $\bar{R}=\int_{\Delta} R d \mu_{0}$. One of the main achievements in [Y2, YY] is the fact that the decay of the tail of return times determines the speed of decay of correlations for Hölder continuous (or Lipschitz) observables. Namely, if $\phi: \mathcal{X} \rightarrow \mathbb{R}$ is Hölder continuous of exponent $0<\iota \leq 1$, with Hölder constant

$$
K_{\iota}(\phi):=\sup _{x \neq y} \frac{|\phi(x)-\phi(y)|}{(\operatorname{dist}(x, y))^{\iota}}
$$

$\psi: \mathcal{X} \rightarrow \mathbb{R}$ is in $L^{\infty}(\mathrm{Leb})$ and the tower has exponential tail, then there are $C>0$ and $\alpha^{\prime}>0$ such that

$$
\left|\int \phi \cdot\left(\psi \circ f^{t}\right) d \mu-\int \phi d \mu \int \psi d \mu\right| \leq C K_{\iota}(\phi)\|\psi\|_{\infty} \mathrm{e}^{-\alpha t}, \quad \forall t \in \mathbb{N}_{0} .
$$

Theorem 6. Let $\mathcal{X}$ be a d-dimensional compact Riemannian manifold and assume that $f: \mathcal{X} \rightarrow \mathcal{X}$ is a $C^{2}$ endomorphism admitting a Young tower with exponential tail. Consider a stochastic process $X_{0}, X_{1}, \ldots$ defined by (1.1) and (1.9), for some choice of $\zeta \in \mathcal{X}$. Then, for Leb-almost every $\zeta \in \mathcal{X}$ chosen, conditions $D_{3}\left(u_{n}\right)$ (or $D_{2}\left(u_{n}\right)$ ) and $D^{\prime}\left(u_{n}\right)$ hold, where $u_{n}$ is a sequence of levels satisfying (1.4).

Together with the results in Section 1.3, Section 3 and Section 4 we get the following corollary.

Corollary 7. Let $\mathcal{X}$ be a d-dimensional compact Riemannian manifold and assume that $f: \mathcal{X} \rightarrow \mathcal{X}$ is a $C^{2}$ endomorphism admitting a Young tower with exponential tail. Consider a stochastic process $X_{0}, X_{1}, \ldots$ defined by (1.1) and (1.9) for some $\zeta \in \mathcal{X}$. Then, for Lebalmost every choice of $\zeta \in \mathcal{X}$, the following assertions hold:

(1) We have an EVL for $M_{n}$, defined in (1.2), which coincides with that one of $\hat{M}_{n}$ defined in (1.5). In particular, it must be of one of the three classical types. Moreover, for every $i \in\{1,2,3\}$, if $g$ is of type $g_{i}$ then we have an $E V L$ for $M_{n}$ of type $E V_{i}$.

(2) We have exponential HTS to balls at $\zeta \in \mathcal{X}$.

(3) The EPP $N_{n}$ defined in (3.3) is such that $N_{n} \stackrel{d}{\rightarrow} N$, as $n \rightarrow \infty$, where $N$ denotes a Poisson Process with intensity 1.

(4) The same applies to the HTPP $N_{n}^{*}$ defined in (3.2).

6.1. Proof of Theorem 6. To show this result, one needs only to realise that Collet's proof of [Col2, Theorem 1] may be mimicked in our multi-dimensional setting with minor adjustments. Thus, instead of repeating all the arguments, we will prove that $D_{3}\left(u_{n}\right)$ and $D^{\prime}\left(u_{n}\right)$ hold just by redoing the parts that need to be adapted to this more general higher 
dimensional setting. However, in order to keep on track we will restate all the Lemmas (with the necessary adjustments) of Collet's proof.

The first lemma is technical and very simple to prove.

Lemma 6.1. For any $v \geq 1$

$$
\sum_{l, R_{l}>v} R_{l} \operatorname{Leb}\left(\Delta_{l}\right) \leq\left\{\begin{array}{l}
2 \sum_{s=v / 2}^{\infty} \operatorname{Leb}(\{R>s\}) \\
\sum_{s=v}^{\infty} \operatorname{Leb}(\{R>s\})+v \operatorname{Leb}(\{R>v\}) .
\end{array}\right.
$$

See [Col2, Lemma 2.1].

Next result gives a relation between the measure $\mu$ of small sets and their respective Lebesgue measure.

Lemma 6.2. Under $\left(\mathbf{Y}_{4}{ }^{2}\right)$, there are two positive constants $C$ and $\theta$ such that for any Lebesgue measurable set $A$, we have

$$
\mu(A) \leq C \operatorname{Leb}(A)^{\theta} .
$$

See [Col2, Lemma 2.2].

To prove condition $D^{\prime}\left(u_{n}\right)$ we need to show that the $\mu$-measure of the set of points $x$ that are too rapidly recurrent is small. For every $v \in \mathbb{N}$ and any $\epsilon>0$ we define the set $\mathcal{E}_{v}(\epsilon)$ of points that come back very close to the initial position after $v$ iterates

$$
\mathcal{E}_{v}(\epsilon)=\left\{x,\left|x-f^{v}(x)\right|<\epsilon\right\} .
$$

Since $\mathcal{X}$ is compact and $f$ is $C^{2}$ we may define $\Upsilon:=\sup \left\{\|D f(x)\|_{\infty} x \in \mathcal{X}\right\}$.

Proposition 6.3. Under $\left(\overline{\left.\mathbf{Y}_{4}{ }^{3}\right)}\right.$, there exist positive constants $C, \alpha^{\prime}$ and $\eta<1$ such that for any integer $v$ and any $\epsilon>0$ we have

$$
\mu\left(\mathcal{E}_{v}(\epsilon)\right) \leq C\left(v^{2} \epsilon^{\eta}+e^{-\alpha^{\prime} v}\right)
$$

Proof. We follow the proof of the corresponding result [Col2, Proposition 2.3] very closely. We will consider the intersection with $\mathcal{E}_{v}(\epsilon)$ of the various cylinders where $f^{v}$ is one-to-one. From (6.1), we have to consider the intersection of these sets with $f^{j}\left(\Delta_{l}\right)$. We will start by choosing a number $1 / 2>\xi>0$ such that $\beta \Upsilon^{\xi}<1$ and assume first that $R_{l}<\xi v$. If we apply $f^{R_{l}-j}$ on $f^{j}\left(\Delta_{l}\right)$, we land in $\Delta$ and we have to apply $f^{v-R_{l}+j}$ to get the image under $f^{v}$. At this point it is convenient to introduce the following construction. Let $\left(s_{j}\right)$ be a sequence of integers. We denote by $\Delta_{s_{1}, s_{2}, \ldots, s_{r}}$ the set

$$
\Delta_{s_{1}, s_{2}, \ldots, s_{r}}=\Delta_{s_{1}} \cap f^{-R_{s_{1}}} \Delta_{s_{2}} \cap f^{-\left(R_{s_{1}}+R_{s_{2}}\right)} \Delta_{s_{3}} \cap \cdots \cap f^{-\left(R_{s_{1}}+\cdots+R_{s_{r-1}}\right)} \Delta_{s_{r}} .
$$

In other words, this is the subset $A$ of $\Delta_{s_{1}}$ which is mapped by $f^{R_{s_{1}}+\cdots+R_{s_{r-1}}}$ bijectively onto $\Delta_{s_{r}}$ with

for $p=1, \ldots, r-1$.

$$
f^{R_{s_{1}}+\cdots+R_{s_{p}}}(A) \subset \Delta_{s_{p+1}}
$$


For fixed $v, l$ and $j$, we now consider all the sets $\Delta_{s_{1}, \ldots, s_{r}}$ with $R_{s_{1}}+\cdots+R_{s_{r-1}}+R_{l}-j<v$ and $R_{s_{1}}+\cdots+R_{s_{r}}+R_{l}-j \geq v$. Together with $\left\{R>v-1-R_{l}+j\right\}$, this gives a partition of $\Delta$. We then construct a partition of $f^{j}\left(\Delta_{l}\right)$ by pulling back this partition by $f^{R_{l}-j}$. We now consider $f^{v}$ on each atom of this partition. Let

$$
A=A_{l, j, s_{1}, \ldots, s_{r}}=f^{j}\left(\Delta_{l}\right) \cap f^{j-R_{l}}\left(\Delta_{s_{1}, \ldots, s_{r}}\right) .
$$

We first assume that $R_{s_{r}}<\xi v$ and $A$ has a 'large' image under $f^{v}$, namely

$$
\left|f^{v}(A)\right| \geq \delta,
$$

where $\delta$ is a positive number to be chosen adequately later on. Let $J:=R_{s_{1}}+\cdots+$ $R_{s_{r}}+R_{l}-j$ and $B:=\mathcal{E}_{v}(\epsilon) \cap A$, which we may assume to be nonempty. We argue that $\left|f^{v}(B)\right|=O\left(\epsilon^{d}\right)$. To see this, let $x, y \in A$ be such that

$$
\operatorname{dist}\left(f^{v}(x), f^{v}(y)\right)=\operatorname{diam}\left(f^{v}(B)\right):=\sup \left\{\operatorname{dist}(z, w): z, w \in f^{v}(B)\right\} .
$$

By $\left(\mathbf{Y}_{2}\right)$, it follows that

$$
\operatorname{dist}(x, y) \leq C \beta^{J} \operatorname{dist}\left(f^{J}(x), f^{J}(y)\right) .
$$

Moreover, by definition of $\Upsilon$, we also have

$$
\operatorname{dist}\left(f^{v}(x), f^{v}(y)\right) \geq \Upsilon^{-J+v} \operatorname{dist}\left(f^{J}(x), f^{J}(x)\right) \geq \Upsilon^{-R_{s_{r}}} \operatorname{dist}\left(f^{J}(x), f^{J}(x)\right) .
$$

Consequently, since by assumption $R_{s_{r}}<\xi v$, we have

$$
\frac{\operatorname{dist}(x, y)}{\operatorname{dist}\left(f^{v}(x), f^{v}(y)\right)} \leq C \beta^{J} \Upsilon^{R_{s_{r}}} \leq O\left(\beta^{v} \Upsilon^{\xi v}\right) \leq O\left(\left(\beta \Upsilon^{\xi}\right)^{v}\right) .
$$

Since $\beta \Upsilon^{\xi}<1$, by the choice of $\xi$, then we may pick $v_{0} \in \mathbb{N}$, only depending on $f$, such that for all $v \geq v_{0}$ we have

$$
\operatorname{dist}(x, y) \leq \frac{1}{2} \operatorname{dist}\left(f^{v}(x), f^{v}(y)\right) .
$$

Observe that for $v<v_{0}$, Proposition 6.3 simply holds with $C$ sufficiently large.

Now, assuming that $x, y \in B \subset \mathcal{E}_{v}(\epsilon)$ we have

$$
\begin{aligned}
\operatorname{dist}\left(f^{v}(x), f^{v}(y)\right) & \leq \operatorname{dist}\left(f^{v}(x), x\right)+\operatorname{dist}(x, y)+\operatorname{dist}\left(y, f^{v}(y)\right) \\
& \leq \epsilon+\frac{1}{2} \operatorname{dist}\left(f^{v}(x), f^{v}(y)\right)+\epsilon .
\end{aligned}
$$

This means that $\operatorname{diam}\left(f^{v}(B)\right) \leq 4 \epsilon$. If $x, y \notin B$ then we could replace them by close enough $x^{\prime}, y^{\prime} \in B$ so that $\operatorname{diam}\left(\overline{f^{v}}(B)\right) \leq 5 \epsilon$. Hence, we have proved that $\left|f^{v}(B)\right|=O\left(\epsilon^{d}\right)$.

Using distortion, we get

$$
|B| /|A|=O\left(\epsilon^{d} / \delta\right),
$$

and

$$
\left|\Delta_{l} \cap f^{-j}(B)\right| /\left|\Delta_{l} \cap f^{-j}(A)\right|=O\left(\epsilon^{d} / \delta\right) .
$$


Since $\mu_{0}$ is equivalent to the Lebesgue measure on $\Delta$, then

$$
\mu_{0}\left(\Delta_{l} \cap f^{-j}(B)\right)=O\left(\frac{\epsilon^{d}}{\delta}\right) \mu_{0}\left(\Delta_{l} \cap f^{-j}(A)\right) .
$$

Next we sum over all sets $A$ as above, contained in $f^{j}\left(\Delta_{l}\right)$ and such that they have 'large' image under $f^{v}$. Since they are disjoint we get a contribution bounded above by $O\left(\epsilon^{d} / \delta\right) \mu_{0}\left(\Delta_{l}\right)$. Summing over $j$ we get a bound $O\left(\epsilon^{d} / \delta\right) R_{l} \mu_{0}\left(\Delta_{l}\right)$. Finally, summing over $l$ we get the estimate: $O\left(\epsilon^{d} / \delta\right)$. This ends the estimate in the good case when segments $A$ reach 'large' scale in $v$ steps.

We next have to gather the estimates for all the left-over bad cases. These bad cases are dealt with by realising that they correspond to large values of $R$, whose tail we are assuming to decay exponentially fast. We skip the study of these cases and refer the reader to [Col2, Proposition 2.3] where they are treated without any particular unidimensional argument.

Finally, collecting all the estimates, there exists $C>0$ so that

$$
\begin{aligned}
\mu\left(\mathcal{E}_{v}(\epsilon)\right)=O\left(\frac{\epsilon^{d}}{\delta}+\sum_{s>\xi v / 2}\right. & \mu_{0}(R>s) \\
& \left.\quad+v \operatorname{Leb}(R \geq(1-\xi) v)+v^{2} \mu(R \geq \xi v)+v^{2} \mu\left(R>C \log \delta^{-1}\right)\right) .
\end{aligned}
$$

Using Lemma 6.2 and $\left(\mathbf{Y}_{4}{ }^{\text {? }}\right.$ ) we have

$$
\mu\left(\mathcal{E}_{v}(\epsilon)\right)=O\left(\frac{\epsilon^{d}}{\delta}+v^{2} e^{-\alpha \theta \xi v}+v^{2} \delta^{\gamma}\right)
$$

for some $1>\gamma>0$. The result follows by taking the minimum with respect to $\delta$.

Let $\left\{E_{v}\right\}_{v \in \mathbb{N}}$ be the sequence of sets defined by

$$
E_{v}=\left\{y: \exists j \in\left\{1, \ldots,(\log v)^{5}\right\},\left|y-f^{j}(y)\right| \leq v^{-1}\right\} .
$$

Corollary 6.4. There exist positive constants $C^{\prime}$ and $\beta^{\prime}<1$ such that for any integer $v$

$$
\mu\left(E_{v}\right) \leq C^{\prime} v^{-\beta^{\prime}} .
$$

See Col2, Corollary 2.4].

We not only need to control the set of points which recur too fast, but also the set of points for which a neighbour recurs too fast. For positive numbers $\psi$ and $\rho$ to be fixed below, we define a sequence of measurable sets $\left\{F_{v}\right\}_{v \in \mathbb{N}}$ by

$$
F_{v}=\left\{x: \mu\left(B_{v^{-\psi}}(x) \cap E_{v^{\psi}}\right) \geq \kappa v^{-(d+\rho) \psi}\right\} .
$$

Lemma 6.5. There exist positive numbers $\rho$ and $\psi$ such that Leb $\left(\bigcap_{i \geq 1} \bigcup_{v \geq i} F_{v}\right)=0$. 
We refer to [Col2, Lemma 2.5] and references therein. The proof uses maximal functions and a result by Hardy and Littlewood which still holds in higher dimensions.

As we have seen in the proof of Corollary 6, it is very easy to show that $D_{3}\left(u_{n}\right)$ holds when we have decay of correlations for observables of bounded variation. However, in this setting, decay of correlations is only available for Hölder continuous functions against $L^{\infty}$ ones, instead (see (6.2)). This means that we cannot use the test function $\phi=\mathbf{1}_{\left\{X_{0}>u_{n}\right\}}$, as we did before. However, proceeding as in [Col2, Lemma 3.3], we use a suitable Hölder approximation and show that the same result follows:

Lemma 6.6. Assume that there exists a rate function $\Theta: \mathbb{N} \rightarrow \mathbb{R}$, such that for every Hölder continuous (or Lipschitz) observable $\phi$ and all $L^{\infty}$ observable $\psi$ we have:

$$
\left|\int \phi \cdot\left(\psi \circ f^{t}\right) d \mu-\int \phi d \mu \int \psi d \mu\right| \leq K_{\iota}(\phi)\|\psi\|_{\infty} \Theta(t), \quad \forall t \in \mathbb{N}_{0} .
$$

Then, for every $\zeta \in \mathcal{X}, 0<s<1, \eta>0$ and all measurable set $W$ we have

$$
\left|\mu\left(B_{s}(\zeta) \cap f^{-t}(W)\right)-\mu\left(B_{s}(\zeta)\right) \mu(W)\right| \leq s^{-(1+\eta)} \Theta(t)+O\left(s^{\theta(d+\eta)}\right),
$$

where $\theta$ is the number given in Lemma 6.2.

Proof. For a fixed $\eta>0$ we build the Hölder approximation $\phi$ of $\mathbf{1}_{B_{s}(\zeta)}$. Let $B:=B_{s}(\zeta)$ and $D:=\overline{B_{s-s^{1+\eta}}(\zeta)}$, where $\bar{A}$ denotes the closure of $A$. Define $\phi: \mathcal{X} \rightarrow \mathbb{R}$ as

$$
\phi(x)= \begin{cases}0 & \text { if } x \notin B \\ \frac{\operatorname{dist}(x, \mathcal{X} \backslash B)}{\operatorname{dist}(x, \mathcal{X} \backslash B)+\operatorname{dist}(x, D)} & \text { if } x \in B \backslash D . \\ 1 & \text { if } x \in D\end{cases}
$$

Observe that $\phi$ is Hölder continuous (Lipschitz) with Hölder constant $s^{-(1+\eta)}$.

Now, we apply the decay of correlations to the Hölder continuous function $\phi$ against $\mathbf{1}_{W} \in$ $L^{\infty}$ to get

$$
\left|\int \phi \cdot\left(\mathbf{1}_{W} \circ f^{t}\right) d \mu-\int \phi d \mu \int \mathbf{1}_{W} d \mu\right| \leq s^{-(1+\eta)} \Theta(t) .
$$

Noticing that the support of $\mathbf{1}_{B}-\phi$ is contained in $B \backslash D$ whose Lebesgue measure is $O\left(s^{d+\eta}\right)$ and using Lemma 6.2 we get

$$
\left|\mu\left(B_{s}(\zeta) \cap f^{-t}(W)\right)-\mu\left(B_{s}(\zeta)\right) \mu(W)\right| \leq s^{-(1+\eta)} \Theta(t)+O\left(s^{\theta(d+\eta)}\right) .
$$

Proof of Theorem 6. First let us show that $D_{3}\left(u_{n}\right)$ holds. Since in this setting we have exponential decay of correlations for Hölder continuous functions (see (6.2) ) and $\left\{X_{0}>\right.$ $\left.u_{n}\right\}=B_{g^{-1}\left(u_{n}\right)}(\zeta)$ then by Lemma 6.6 we may take

$$
\gamma(n, t)=O\left(\left(g^{-1}\left(u_{n}\right)\right)^{-1-\eta} \mathrm{e}^{-\alpha t}+\left(g^{-1}\left(u_{n}\right)\right)^{\theta(d+\eta)}\right) .
$$


Hence, recalling that $g^{-1}\left(u_{n}\right) \sim\left(\frac{\tau}{\kappa \rho(\zeta) n}\right)^{1 / d}$, if we consider $t_{n}=\sqrt{n}$, for example, and choose $\eta$ from Lemma 6.6 so that $\theta(d+\eta) / d>2$ (where $\theta$ is given by Lemma 6.2), then we easily get that $n \gamma\left(n, t_{n}\right) \underset{n \rightarrow \infty}{\longrightarrow} 0$ which gives $D_{3}\left(u_{n}\right)$.

Now, it only remains to show that $D^{\prime}\left(u_{n}\right)$ also holds. Recall the stochastic process $X_{0}, X_{1}, \ldots$ given by (1.1) for observables defined by (1.9), achieving a global maximum at $\zeta \in \mathcal{X}$. At this point, we describe the full Lebesgue measure set of points $\zeta \in \mathcal{X}$ for which Theorem 6 holds. We take $\zeta$ for which Lebesgue's differentiation theorem holds (with respect to the measure $\mu$ ) and $\zeta \in \cup_{i \geq 1} \cap_{j \geq i} \mathcal{X} \backslash F_{j}$, which by Lemma 6.5 is also a full Lebesgue measure set. For each such $\zeta$, let $v_{0}(\zeta) \in \mathbb{N}$ be such that $\zeta \notin F_{j}$ for all $j \geq v_{0}(\zeta)$.

We consider a turning instant $t=t(n)=\left\lfloor(\log n)^{2}\right\rfloor$, and split the sum in $D^{\prime}\left(u_{n}\right)$ into the period before $t$ and after $t$.

For the later we use exponential decay of correlations (6.2) and Lemma 6.6 to get, for some $C>0$,

$$
\begin{aligned}
S_{2}(t, n, k) & :=n \sum_{j=t}^{\lfloor n / k\rfloor} \mu\left(\left\{X_{0}>u_{n}\right\} \cap\left\{X_{j}>u_{n}\right\}\right) \\
& \leq n\left\lfloor\frac{n}{k}\right\rfloor \mu\left(X_{0}>u_{n}\right)^{2}+n\left\lfloor\frac{n}{k}\right\rfloor\left(g^{-1}\left(u_{n}\right)\right)^{\theta(d+\eta)}+n\left\lfloor\frac{n}{k}\right\rfloor\left(g^{-1}\left(u_{n}\right)\right)^{-1-\eta} C \mathrm{e}^{-\alpha t} .
\end{aligned}
$$

Recalling that $\mu\left(X_{0}>u_{n}\right) \sim \tau n^{-1}$ and $g^{-1}\left(u_{n}\right) \sim\left(\frac{\tau}{\kappa \rho(\zeta) n}\right)^{1 / d}$, we have

$$
S_{2}(t, n, k)=O\left(\frac{1}{k}+\frac{n^{2}}{k} n^{-\theta(d+\eta) / d}+\frac{n^{2}}{k} n^{(1+\eta) / d} \mathrm{e}^{-\alpha^{\prime} \log ^{2}(n)}\right) .
$$

So, if we chose $\eta$ so that $\theta(d+\eta) / d>2$ then $\lim _{k \rightarrow \infty} \limsup _{n \rightarrow \infty} S_{2}(t, n, k)=0$.

We are left with the first period from 1 to $t$ and the respective sum

$$
S_{1}(t, n):=n \sum_{j=1}^{t} \mu\left(\left\{X_{0}>u_{n}\right\} \cap\left\{X_{j}>u_{n}\right\}\right) .
$$

We set $v=v(n)=\left\lfloor\left(3 g^{-1}\left(u_{n}\right)\right)^{-1 / \psi}\right\rfloor$, where $\psi$ is given in Lemma 6.5, Observe that

$$
\left\{X_{0}>u_{n}\right\}=B_{g^{-1}\left(u_{n}\right)}(\zeta) \subset B_{v^{-\psi}}(\zeta)
$$

and, if $y \in\left\{X_{0}>u_{n}\right\} \cap\left\{X_{j}>u_{n}\right\}$, then

$$
\operatorname{dist}\left(f^{j}(y), y\right) \leq \operatorname{dist}\left(f^{j}(y), \zeta\right)+\operatorname{dist}(\zeta, y) \leq 2 g^{-1}\left(u_{n}\right)<v^{-\psi},
$$

which implies that

$$
\left\{X_{0}>u_{n}\right\} \cap\left\{X_{j}>u_{n}\right\} \subset B_{v^{-\psi}}(\zeta) \cap E_{v^{\psi}} .
$$


We take $n$ so large that $v=v(n) \geq v_{0}(\zeta)$. Hence $\zeta \notin F_{v}$. Using (6.4), the definition of $F_{v}$ and the fact $g^{-1}\left(u_{n}\right) \sim\left(\frac{\tau}{\kappa \rho(\zeta) n}\right)^{1 / d}$, we have

$$
\mu\left(\left\{X_{0}>u_{n}\right\} \cap\left\{X_{j}>u_{n}\right\}\right)=O\left(v^{-\psi(d+\rho)}\right)=O\left(n^{-(d+\rho) / d}\right) .
$$

Hence, $\limsup _{n \rightarrow \infty} S_{1}(t, n) \leq \limsup _{n \rightarrow \infty} O\left(n \log ^{2}(n) n^{-(d+\rho) / d}\right)=0$.

6.2. An Example. Here we present a $C^{1}$ open class of local diffeomorphisms with no critical points that are non-uniformly expanding in the sense of [ABV, Al]. Namely, let $f: M \rightarrow M$ be a $C^{1}$ local diffeomorphism, we say that $f$ is non-uniformly expanding if there exists a constant $\lambda>0$ such that for Lebesgue almost all points $x \in M$ the following non-uniform expansivity condition is satisfied:

$$
\liminf _{n \rightarrow \infty} \frac{1}{n} \sum_{i=0}^{n-1} \log \left\|D f_{f^{i}(x)}^{-1}\right\|^{-1} \geq \lambda>0 .
$$

Condition (6.5) implies that the expansion time function

$$
\mathcal{E}(x)=\min \left\{N: \frac{1}{n} \sum_{i=0}^{n-1} \log \left\|D f_{f^{i}(x)}^{-1}\right\|^{-1} \geq \lambda / 2 \quad \forall n \geq N\right\}
$$

is defined and finite almost everywhere in $M$. We think of this as the waiting time before the exponential derivative growth kicks in. We are now able to define the Hyperbolic tail set, at time $n \in \mathbb{N}$,

$$
\Gamma_{n}=\{x \in I: \mathcal{E}(x)>n\},
$$

which can be seen as the set of points that at time $n$ have not reached a satisfactory exponential growth of the derivative. Applying ALP and G2 together shows that these maps admit a Young tower whose return time tail is related to the volume decay rate of the hyperbolic tail set.

The class we consider here is obtained by deformation of a uniformly expanding map by isotopy inside some small region. In general, these maps are not expanding: deformation can be made in such way that the new map has periodic saddles. We follow the construction in $\mathrm{ABV}, \mathrm{Al}$.

Let $M$ be any compact Riemannian $d$-dimensional manifold supporting some uniformly expanding map $f_{0}$ : there exists $\sigma_{0}>1$ such that $\left\|D f_{0}(x) v\right\|>\sigma_{0}\|v\|$ for every $x \in M$ and every $v \in T_{x} M$. Let $V \subset M$ be small compact domain, so that $\left.f_{0}\right|_{V}$ is one-to-one. Let $f_{1}$ be a $C^{1}$ map coinciding with $f_{0}$ in $M \backslash V$ for which the following holds:

(1) $f_{1}$ is volume expanding everywhere: there is $\sigma_{1}>1$ such that

$$
\left|\operatorname{det} D f_{1}(x)\right|>\sigma_{1}, \quad \text { for every } x \in M \text {; }
$$

(2) $f_{1}$ is not too contracting on $V$ : there is small $\delta>0$ such that

$$
\left\|D f_{1}(x)^{-1}\right\|<1+\delta, \quad \text { for every } x \in V \text {. }
$$


We consider the class of maps $f$ in a small $C^{1}$-neighbourhood $\mathcal{F}$ of $f_{1}$.

In [Al, Section 6] it was shown that these maps satisfy condition (6.5) and there exist $C, \gamma>0$ such that $\operatorname{Leb}\left(\Gamma_{n}\right) \leq C \mathrm{e}^{-\gamma n}$ for all $n \in \mathbb{N}$. Now, using the results in [G2] this

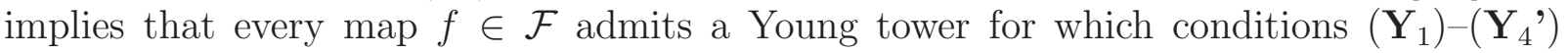
are satisfied. This means that we can apply Theorem [6 and obtain that all assertions of Corollary 7 hold for this class of maps $\mathcal{F}$.

\section{REFERENCES}

[Ab] M. Abadi, Sharp error terms and necessary conditions for exponential hitting times in mixing processes, Ann. Probab. 32 (2004) 243-264.

[AG] M. Abadi, A. Galves, Inequalities for the occurrence times of rare events in mixing processes. The state of the art, Markov Process. Related Fields 7 (2001) 97-112.

[Al] J.F. Alves, Strong statistical stability of non-uniformly expanding maps, Nonlinearity 17 (2004) 1193-1215.

[ABV] J.F. Alves, C. Bonatti, M. Viana, SRB measures for partially hyperbolic systems whose central direction is mostly expanding, Invent. Math. 140 (2000) 351-398.

[ALP] J.F. Alves, S. Luzzatto, V. Pinheiro, Markov structures and decay of correlations for nonuniformly expanding dynamical systems, Ann. Inst. H. Poincaré Anal. Non Linéaire 22 (2005) 817-839.

[BC] M. Benedicks and L. Carleson, On iterations of $1-a x^{2}$ on $(-1,1)$, Ann. Math. 122 (1985), 1-25.

[BRSS] H. Bruin, J. Rivera-Letelier, W. Shen, S. van Strien, Large derivatives, backward contraction and invariant densities for interval maps, Invent. Math. 172 (2008) 509-593.

[BSTV] H. Bruin, B. Saussol, S. Troubetzkoy, S. Vaienti, Return time statistics via inducing, Ergodic Theory Dynam. Systems 23 (2003) 991-1013.

[BT] H. Bruin, M. Todd, Return time statistics for invariant measures for interval maps with positive Lyapunov exponent, arXiv:0708.0379.

[BV] H. Bruin, S. Vaienti, S. Return time statistics for unimodal maps, Fund. Math. 176 (2003) 77-94.

[C1] Z. Coelho, Asymptotic laws for symbolic dynamical systems, Topics in symbolic dynamics and applications (Temuco 1997) 123-165, LMS Lecture Notes Series 279 Cambridge Univ. Press, 2000 .

[C2] Z. Coelho, The loss of tightness of time distributions for homeomorphisms of the circle, Trans. Amer. Math. Soc. 356 (2004) 4427-4445

[CF] Z. Coelho, E. de Faria, Limit laws of entrance times for homeomorphisms of the circle, Israel J. Math. 93 (1996) 93-112.

[Col1] P. Collet, Some ergodic properties of maps of the interval, Dynamical Systems (Temuco, 1991/1992), (Travaux en cours, 52). Herman, Paris, 1996, pp. 5591

[Col2] P. Collet, Statistics of closest return for some non-uniformly hyperbolic systems, Ergodic Theory Dynam. Systems 21 (2001) 401-420.

[DGS] M. Denker, M. Gordin, A.A. Sharova, Poisson limit theorem for toral automorphisms, Illinois J. Math. 48 (2004) 1-20.

[Do] D. Dolgopyat, Limit theorems for partially hyperbolic systems, Trans. Amer. Math. Soc. 356 (2004) 1637-1689

[DM] E.J. Dudewicz, S.N. Mishra, Modern Mathematical Statistics, Wiley Series in Probability and Mathematical Statistics, John Wiley \& Sons, 1988.

[Fe] W. Feller, An introduction to Probability Theory and its Applications, Volume I. Wiley Publications in Statistics, 1952. 
[FF1] A.C.M. Freitas, J.M. Freitas, Extreme values for Benedicks Carleson maps, To appear in Ergodic Theory Dynam. Systems.

[FF2] A.C.M. Freitas, J.M. Freitas, On the link between dependence and independence in Extreme Value Theory for Dynamical Systems, Stat. Probab. Lett. 78 (2008) 1088-1093.

[G1] S. Gouëzel, Sharp polynomial estimates for the decay of correlations, Israel J. Math. 139 (2004) 29-65.

[G2] S. Gouëzel, Decay of correlations for nonuniformly expanding systems, Bull. Soc. Math. France 134 (2006) 1-31.

[HLV] N. Haydn, Y. Lacroix, S. Vaienti, Hitting and return times in ergodic dynamical systems, Ann. Probab. 33 (2005) 2043-2050.

[H] M. Hirata, Poisson law for Axiom A diffeomorphisms, Ergodic Theory Dynam. Systems 13 (1993) 533-556.

[HSV] M. Hirata, B. Saussol, S. Vaienti, Statistics of return times: a general framework and new applications, Comm. Math. Phys. 206 (1999) 33-55.

[HHL] T. Hsing, J. Hüsler, M.R. Leadbetter, On the exceedance point process for a stationary sequence, Probab. Theory Related Fields 78 (1988) 97-112.

[Ka] O. Kallenberg, Random measures, Academic Press Inc., New York, 1986.

[KN] G. Keller and T. Nowicki, Spectral theory, zeta functions and the distribution of periodic points for Collet-Eckmann maps, Comm. Math. Phys. 149 (1992) 31-69.

[LLR] G. Lindgren, M.R. Leadbetter, and H. Rootzén, Extremes and related properties of random sequences and processes, Springer Series in Statistics, Springer-Verlag, New York-Berlin, 1983, XII.

[Pi] B. Pitskel, Poisson limit law for Markov chains, Ergodic Theory Dynam. Systems 11 (1991) 501-513.

[R] M. Rychlik, Bounded variation and invariant measures, Studia Math. 76 (1983) 69-80.

[Y1] L. S. Young, Decay of correlations for certain quadratic maps, Comm. Math. Phys. 146 (1992) 123-138.

[Y2] L.S. Young, Statistical properties of dynamical systems with some hyperbolicity, Ann. of Math. (2) 147 (1998) 585-650.

[Y3] L.S. Young, Recurrence times and rates of mixing, Israel J. Math. 110 (1999) 153-188.

Ana Cristina Moreira Freitas, Centro de Matemática \& Faculdade de Economia da Universidade do Porto, Rua Dr. Roberto Frias, 4200-464 Porto, Portugal

E-mail address: amoreira@fep.up.pt

Jorge Milhazes Freitas, Centro de Matemática da Universidade do Porto, Rua do Campo Alegre 687, 4169-007 Porto, Portugal

E-mail address: jmfreita@fc.up.pt

$U R L:$ http://www.fc.up.pt/pessoas/jmfreita

Mike Todd, Centro de Matemática da Universidade do Porto, Rua do Campo Alegre 687, 4169-007 Porto, Portugal

E-mail address: mtodd@fc.up.pt

$U R L:$ http://www.fc.up.pt/pessoas/mtodd 